\title{
CONSERVATIVITY OF HEYTING IMPLICATION OVER RELEVANT QUANTIFICATION
}

\author{
ROBERT GOLDBLATT \\ Centre for Logic, Language and Computation, Victoria University of Wellington
}

\begin{abstract}
It is known that propositional relevant logics can be conservatively extended by the addition of a Heyting (intuitionistic) implication connective. We show that this same conservativity holds for a range of first-order relevant logics with strong identity axioms, using an adaptation of Fine's stratified model theory. For systems without identity, the question of conservatively adding Heyting implication is thereby reduced to the question of conservatively adding the axioms for identity. Some results in this direction are also obtained. The conservative presence of Heyting implication allows the development of an alternative model theory for quantified relevant logics.
\end{abstract}

§1. Introduction and overview. This paper shows that various systems of first-order logic with relevant implication can be conservatively extended by the addition of a Heyting implication. By the latter we mean a binary connective $\supset$ that relates to the conjunction connective $\wedge$ by the deduction principle

$$
A \wedge B \vdash C \quad \text { iff } \quad A \vdash B \supset C
$$

of " $\wedge$-Residuation". In this context, a consecution relation $A \vdash B$ asserts that the relevant implication $A \rightarrow B$ is a theorem. ${ }^{1}$

Given some deduction system $\mathrm{L}$, defined by specified axioms and rules in a particular language without $\supset$, we call HL the system having those axioms and rules of $\mathrm{L}$ together with the rule (1.1), all stated for formulas in the expanded language containing $\supset$. HL is called conservative over $\mathrm{L}$ if it has no new $\supset$-free theorems: every $\supset$-free formula that is a theorem of HL must already be an L-theorem.

We will prove that conservativity of $\mathrm{HL}$ over $\mathrm{L}$ holds when $\mathrm{L}$ is a certain extension of the Anderson-Belnap system RQ by axioms for an identity predicate $\approx$, as well for many subsystems of this one obtained by varying the underlying propositional axioms. These results are then extended to systems with contrapositive negation, by which we mean systems that may lack the double-negation elimination postulate $\neg \neg A \rightarrow A$, and in which the quantifiers $\forall, \exists$, and the propositional connectives $\wedge, \vee, \rightarrow, \neg, \circ$ (fusion), are all independent. All of this requires new completeness theorems giving semantic characterizations of the logics involved. For the logics without double-negation elimination we use a binary incompatibility relation $\perp$ to interpret negation, a modeling that goes back to an early paper of the author (Goldblatt, 1974).

For all the systems just mentioned, the question of conservatively adding $\supset$ is thereby reduced to the problem of conservatively adding axioms for identity. In the last part of the

Received: December 5, 2008

1 So $A \vdash B$ does not mean that $A \supset B$ is a theorem. 
paper we give some results in this direction for the $\{\forall, \wedge, \vee, \rightarrow, \neg, \circ\}$-fragment of many contrapositive-negation systems. The final Section 8 contains a full summary of all the semantic characterizations and conservativity results proved in the paper.

These results are not just a series of technical curiosities. The conservative presence of $\supset$ has a significant impact on the properties of canonical models used in completeness proofs, and has allowed the development of a new kind of model theory for quantified relevant logics by adapting ideas from the Kripke-Joyal semantics for intuitionistic logic. Details of this will be reported elsewhere.

It is already known that $\supset$ can be conservatively added to the propositional relevant logic $\mathrm{R}$ (and many of its subsystems). This observation is due to Restall (1998), using a strikingly direct argument based on the completeness of $\mathrm{R}$ with respect to the relational models of Routley and Meyer (1973). If a $\supset$-free propositional formula $A$ is not an R-theorem, then it is falsified by some Routley-Meyer model $\mathfrak{A}$. This model has a partial order $\geq$ that can be used to interpret $\supset$ by Kripke's clause for satisfaction of intuitionistic implication:

$$
t \models B \supset C \quad \text { iff } \quad \text { for all } u \geq t, u \models B \text { implies } u \models C .
$$

$\mathfrak{A}$ is thereby expanded to a model $\mathfrak{A}^{\prime}$ for the language including $\supset$. The interpretation of $A$ is unchanged, so $\mathfrak{A}^{\prime}$ still falsifies $A$. It suffices then to show that $\mathfrak{A}^{\prime}$ is an HR-model, in order to conclude that $A$ is not an HR-theorem. The clause (1.2) ensures that (1.1) is sound in $\mathfrak{A}^{\prime}$, so the issue comes down to the $\mathfrak{A}^{\prime}$-soundness of the axioms and rules of $\mathrm{R}$ in the extended language. For this we need the general fact that the truth relation is extendable along the ordering $\geq$, in the sense that for any formula $B$,

$$
t \models B \text { and } u \geq t \text { implies } u \models B .
$$

Now (1.3) is shown by induction on the formation of $B$, with the inductive cases for the R-connectives being just as in Routley and Meyer (1973), and the inductive case that $B$ is $B_{1} \supset B_{2}$ following directly from (1.2) by transitivity of $\geq$, as is well known. That completes the conservativity proof. ${ }^{2}$

This argument suggests that a similar proof should work for the first-order logic RQ, by invoking its completeness with respect to the partially ordered stratified models of Fine (1988). But here there is an obstacle, concerning the proof that the expanded structure $\mathfrak{A}^{\prime}$ is an RQ-model. Soundness of the quantifier axioms depends, not just on the $\geq$-extendability of truth (1.3), but on several other results proven by induction on formula formation. Two of these, the Truth Across and Truth Down lemmas, are problematic for the inductive case of $\supset$.

It turns out that Truth Down can be handled by introducing an additional property of stratified models, concerning the down operator $t \mapsto t \downarrow$. This property states that

$$
u \geq t \downarrow \text { implies } \exists u^{+} \geq t\left(u^{+} \downarrow=u\right),
$$

and makes $\downarrow$ into a p-morphism relative to $\geq$, leading to a verification that $t \downarrow \models B \supset C$ iff $t \models B \supset C$. Canonical models for RQ and related logics can be shown to satisfy (1.4)

2 This proof could also be given using the R-models of Fine (1974). A more structural version of the argument observes that the set of propositions (i.e. subsets closed under $\geq$-extension) of an R-model is closed under the implication operator corresponding to (1.2), and so "we do not gain any new propositions on a frame by enriching our expressive powers to include intuitionistic implication" (Restall, 1998, p. 185). 
with the help of the Conjunctive Existential Distribution postulate

$$
A \wedge \exists x B \rightarrow \exists x(A \wedge B),
$$

where $x$ is not free in $A$, and without any use of $\supset$ (see Theorem 4.2 below).

Truth Across, however, does not seem amenable to such a strategy. To explain this, note that a point $t$ in a stratified model can be intuitively thought of as a theory, a collection of propositions closed under whatever those propositions commit one to, or perhaps as a collection of sentences closed under entailment. Each theory has a domain of individuals over which quantified variables range. Given distinct individuals $i, j$ in the domain of $t$, there is a theory ${ }^{i j} \vec{t}$ (" $t$ across"), thought of as the minimal extension of $t$ in which $i$ and $j$ are treated as logically indistinguishable. If $t={ }^{i j} \vec{t}$, then $t$ is called $i j$-symmetric. The Truth Across Lemma asserts that if $t$ is $i j$-symmetric, then $t \models A$ iff $t \models A^{\prime}$, where $A^{\prime}$ is any sentence obtained from $A$ by interchanging some occurrences of the constants $i$ and $j$. But for this to hold inductively when $A$ is $B \supset C$, it would seem to be required that $i j$ symmetry be preserved by $\geq$-extension, that is that if $u \geq t={ }^{i j} \vec{t}$, then $u={ }^{i j} \vec{u}$. It is not evident that this condition can be imposed on general stratified RQ-models.

However, this condition can be imposed for languages that have an identity predicate $\approx$, producing sentences $i \approx j$ in the object language asserting the indistinguishability of $i$ and $j$. If we require that models satisfy

$$
t={ }^{i j} \vec{t} \quad \text { iff } \quad t \models i \approx j,
$$

then $\geq$-extendability of truth ensures $\geq$-extendability of $i j$-symmetry, allowing the Truth Across Lemma to be proved for the case of $\supset$. We use a semantics for identity adapting that of Mares (1992), interpreting $\approx$ as a binary relation $\approx_{t}$ on the domain of individuals associated with each theory $t$. The resulting logic is axiomatized as a system $\mathrm{RQ}^{\approx}$ that has $\mathrm{HRQ} \approx$ as a conservative extension.

There is a subtle technicality in our analysis that is worth pointing out from the outset, since it determines the nature of our canonical model constructions and choice of axioms. The stratified models of Fine (1988) admit empty theories containing no propositions and making no commitments. In particular, in the canonical models used in completeness proofs, at each level of the stratification there is a $\geq$-minimum element satisfying no sentences. These minima are co-theories of $\geq$-maximum elements that themselves satisfy all sentences. Now an empty theory would be $i j$-symmetric but not satisfy $i \approx j$, so would violate (1.5). Therefore we have to eliminate such elements. At the same time we need to keep the maximum elements, as these may be produced by the fusion $t \cdot u$ of two theories $t$ and $u$, an operation used to model relevant implication. But then we have to remove the maximum elements from the domain of the co-theory function, this domain being the set of saturated theories. Thus we do not count maximum theories as saturated, so the whole canonical model construction of Fine (1988) has to be adapted to work under this restriction.

This adaptation requires us to include as axioms the schemas $A \rightarrow \mathbf{v} \approx \mathbf{v}$ (corresponding to reflexivity of all relations $\left.\approx_{t}\right)$ and $\mathbf{v} \approx \mathbf{v} \rightarrow(\mathbf{v} \not \approx \mathbf{v} \rightarrow A)$. The first ensures that all self-identity statements are provably equivalent: $\vdash \mathbf{v} \approx \mathbf{v} \leftrightarrow \mathbf{w} \approx \mathbf{w}$. These axioms appear to violate the principle of relevance of implication. But a liberal relevantism might concede that an assertion $\mathbf{v} \approx \mathbf{v}$ of self-identity is equivalent to the "Verum" $\mathrm{T}$, a weakest proposition that is true if any proposition is, while its negation $\mathbf{v} \not \approx \mathbf{v}$ is equivalent to the "Falsum" F, a strongest proposition implying all others. A theory that treats self-identity literally is committed to the presence of formulas that play the role of $\mathrm{T}$ and $\mathrm{F}$. In any case, 
models satisfying (1.5) validate the axioms $A \rightarrow \mathbf{v} \approx \mathbf{v}$ and $\mathbf{v} \approx \mathbf{v} \rightarrow(\mathbf{v} \not \approx \mathbf{v} \rightarrow A)$, as we show in Corollary 3.2, and these axioms are in turn needed to axiomatize the logic of such models.

Here is a brief summary of the paper. Section 2 reviews Fine's stratified models and the logics they characterize. Section 3 introduces the identity axioms and their models, and gives completeness theorems for logics with these axioms by a canonical model construction. Section 4 gives a semantic analysis of the Conjunctive Existential Distribution postulate, and Section 5 proves the conservativity of Heyting implication over the identity logics of Section 3. Section 6 reworks the whole theory for logics with weaker negation under the $\perp$ interpretation. Section 7 uses algebraic semantics and the ideal-completion of an algebraic model to give conservativity results for adding identity, and for adding Heyting implication, to certain logics with weak negation. Section 8 catalogues all the semantic characterizations and conservativity results shown in the paper.

§2. Stratified models and their logics. Familiarity is assumed with the papers (Fine, 1974, 1988), which will be followed closely, and will be referred to as MFE and SQL. First we review the semantics of SQL, which is based on $\mathfrak{L}$-structures, where $\mathfrak{L}$ is some set of predicates. These structures have the form

$$
\mathfrak{A}=(T, S, D, l, \cdot,-, \geq, \uparrow, \downarrow, \rightarrow, \varphi),
$$

fulfilling the following description:

(i) $T$ (theories) is a set, with members denoted by the letters $t, u, v$, as well as decorations of these like $t^{+}, t^{\prime}, t^{*}$, and so forth.

(ii) $S$ (saturated theories) is a subset of $T$, with members denoted by $a, b, c$, as well as $a^{+}, a^{\prime}$, and so forth.

(iii) $D$ (relative domain) is a function from $T$ to sets, taking each $t \in T$ to its ontology or domain of individuals $D_{t}$. We use $\mathfrak{D}$ (domains) for $\left\{D_{t}: t \in T\right\}$, and $I$ for $\cup \mathfrak{D}$. The members of $I$ may be thought of as constants, so the members of $\mathfrak{D}$ represent various levels of language differing only in the constants they contain. We write $\alpha, \beta, \gamma$ for members of $\mathfrak{D}$, and $i, j, k$ for members of $I$.

The domain equivalence relation $\equiv$ is defined to be $\left\{(t, u) \in T \times T: D_{t}=D_{u}\right\}$. Thus members of the equivalence class $\{u: t \equiv u\}$ all have the same level. ${ }^{3}$

(iv) $l$ (logics) is a function from $\mathfrak{D}$ to $T$, with $D_{l(\alpha)}=\alpha \cdot l(\alpha)$ is thought of as the logic appropriate to $\alpha$. Often this is written just as $l$, with the $\alpha$ omitted.

(v) $\cdot$ (fusion) is a partial binary operation on $T$, with $t \cdot u$ defined only when $t \equiv u$, and having $t \cdot u \equiv t \equiv u$. Usually $t \cdot u$ is written $t u$.

(vi) - (the co-theory operation) is a unary operation on $S$, having $-a \equiv a$. We may write $t-a$ for $t \cdot-a$.

(vii) $\geq$ is a binary relation on $T$, with $t \geq u$ only if $t \equiv u$. The converse of $\geq$ is written $\leq$.

(viii) $\uparrow$ (the up operator) is a partial function from $T \times \mathfrak{D}$ to $T$, with $\uparrow(t, \alpha)$ defined only when $D_{t} \subseteq \alpha$ and having $D_{\uparrow(t, \alpha)}=\alpha$. Usually $\uparrow(t, \alpha)$ is written $t \uparrow^{\alpha}$, or just as $t \uparrow$ if no ambiguity results. It may be thought of as the expansion of theory

${ }^{3}$ In $\mathrm{SQL} \approx$ is used in place of $\equiv$, but here we are reserving $\approx$ as an identity symbol. 
$t$ to $\alpha$, the consequences in the language of $\alpha$ of the theorems of $t$. We also write $t \uparrow^{u}$ for $t \uparrow^{D u}$.

(ix) $\downarrow$ (the down operator) is a partial function from $T \times \mathfrak{D}$ to $T$, with $\downarrow(t, \alpha)$ defined only when $\alpha \subseteq \mathfrak{D}_{t}$ and having $D_{\downarrow(t, \alpha)}=\alpha$. Usually $\downarrow(t, \alpha)$ is written $t \downarrow_{\alpha}$, or just as $t \downarrow$ if no ambiguity results. It may be thought of as the contraction of theory $t$ to $\alpha$, the theorems of $t$ that belong to the language of $\alpha$. We also write $t \downarrow_{u}$ for $t \downarrow_{D u}$.

(x) $\rightarrow$ (the across operator) is a partial function from $T \times\{\{i, j\} \subseteq I: i \neq j\}$ into $T$. $\rightarrow(t,\{i, j\})$ is defined for $i \neq j$ just in case $i, j \in D_{t}$, and is $\equiv$-equivalent to $t$. It is usually written ${ }^{i j} \vec{t}$, or just $\vec{t}$ if no ambiguity results. An element of the form $i j \vec{t}$ is said to be $i j$-symmetric. Note that ${ }^{i j} \vec{t}={ }^{j i} \vec{t}$.

(xi) $\varphi$ (valuation) is a relation holding between a theory $t \in T$ and an $(n+1)$-tuple $\left(R, i_{1}, \ldots, i_{n}\right)$ consisting of an $n$-ary predicate $R \in \mathfrak{L}$ and individuals $i_{1}, \ldots, i_{n}$ from $D_{t}$.

Such a structure will be called a stratified model if it satisfies the following conditions (remember that $t, u, v \in T$ while $a, b, c \in S$ ):

\section{Standard}

(i) $\geq$ is a partial ordering.

(ii) $t \geq u$ implies $(t v \geq u v)$ and ( $v t \geq v u$ ).

(iii) $a \geq t u$ implies $\exists b \geq t(a \geq b u)$ and $\exists b \geq u(a \geq t b)$.

(iv) $l t=t$.

(v) $--a=a$.

(vi) $a \geq b$ implies $-b \geq-a$.

(vii) $\varphi t\left(R, i_{1}, \ldots, i_{n}\right)$ iff $(\forall a \geq t) \varphi a\left(R, i_{1}, \ldots, i_{n}\right)$, for $i_{1}, \ldots, i_{n} \in D_{t}$.

\section{Levels}

(i) $\forall \alpha \exists \beta(\alpha \subset \beta)$.

(ii) $\forall \alpha, \beta \exists \gamma(\alpha \cup \beta \subseteq \gamma)$.

(iii) $\forall \alpha, \beta, \gamma(\alpha \subseteq \beta \subseteq \gamma$ implies $\alpha \cup(\gamma-\beta) \in \mathfrak{D})$.

\section{Behavior of $\uparrow$ and $\downarrow$}

(i) (a) $t \leq u$ implies $t \uparrow \leq u \uparrow$.

(b) $t \leq u$ implies $t \downarrow \leq u \downarrow$.

(ii) (a) $t \uparrow \uparrow=t \uparrow$

(b) $t \downarrow \downarrow=t \downarrow$.

(iii) (a) $t \uparrow \downarrow=t$.

(b) $t \downarrow \uparrow \leq t$.

(c) $t \downarrow \uparrow=t \uparrow \downarrow$, as long as $D_{t} \cap D_{t \downarrow \uparrow}=D_{t \downarrow}$.

(iv) $\varphi t \downarrow\left(R, i_{1}, \ldots, i_{n}\right)$ iff $\varphi t\left(R, i_{1}, \ldots, i_{n}\right)$, for $i_{1}, \ldots, i_{n} \in D_{t \downarrow}$.

\section{Interaction of $\uparrow$ and $\downarrow$}

(i) (With $S$ )

(a) $a \downarrow \in S$.

(b) $a \leq b \downarrow$ implies $\exists a^{+} \leq b\left(a^{+} \downarrow=a\right)$. 
(c) $a \geq t \downarrow$ implies $\exists a^{+} \geq t\left(a^{+} \downarrow \leq a\right)$.

(ii) (With -$)(-a) \downarrow=-(a \downarrow)$.

(iii) (With $\cdot$ )

(a) $(t u) \uparrow=t \uparrow u \uparrow$.

(b) $(t u \uparrow) \downarrow \leq t \uparrow u$.

(iv) (With $l$ ) $l \uparrow=l$, that is for $\alpha \subseteq \beta, l(\alpha) \uparrow^{\beta}=l(\beta)$.

\section{Behavior of $\rightarrow$}

(i) $t \leq \vec{t}$.

(ii) $t \leq u$ implies $\vec{t} \leq \vec{u}$.

(iii) $\overrightarrow{\vec{t}} \leq \vec{t}$.

(iv) $t={ }^{i j} \vec{t}$ and $t \leq a$ implies $\exists a^{\prime}\left(a^{\prime}={ }^{i j} \overrightarrow{a^{\prime}}\right.$ and $\left.t \leq a^{\prime} \leq a\right)$.

(v) $\overrightarrow{(-\vec{a})} \leq-\vec{a}$.

(vi) $\overrightarrow{(t u)} \leq \vec{t} \vec{u} \leq \vec{t} u$.

(vii) $\overrightarrow{(t \uparrow)}=(\vec{t}) \uparrow$.

(viii) $\overrightarrow{(t \uparrow)} \downarrow \leq t$, where $\rightarrow$ is ${ }^{i j} \rightarrow, i \in D_{t \uparrow}-D_{t}$, and $j \in D_{t}$.

(ix) $\varphi t\left(R, i_{1}, \ldots, i_{n}\right)$ implies $\varphi \vec{t}\left(R, i_{1}^{\prime}, \ldots, i_{n}^{\prime}\right)$, where $\rightarrow$ is ${ }^{j k} \rightarrow$ for distinct $j$, $k \in D_{t}$ and, for $p \leq n, i_{p}^{\prime}=i_{p}$ if $i_{p} \notin\{j, k\}$, and $i_{p}^{\prime} \in\{j, k\}$ if $i_{p} \in\{j, k\}$.

Explanation and interpretation of these conditions are given in SQL.

We now explain how a model interprets $\mathfrak{L}$-formulas, constructed from a given set $\mathfrak{L}$ of predicates by the connectives $\wedge, \neg$, and $\rightarrow$ and the universal quantifier $\forall$. The disjunction $A \vee B$ is defined to be $\neg(\neg A \wedge \neg B)$, and $\exists x$ to be $\neg \forall x \neg$. A typographical distinction is made between free and bound (or bindable) individual variables. We assume an infinite supply of real variables $\mathbf{v}, \mathbf{w}$, and an infinite supply of apparent variables $x, y$. Real variables are never bound by $\forall$, so occur only freely. Apparent variables can have both free and bound occurrences. A real formula is one which has no free occurrence of an apparent variable, that is all apparent occurrences are bound, and so any free occurrences are real. We write $\operatorname{Var}(A)$ for the set of real variables occurring in $A$, and if $V$ is a set of real variables, let $\operatorname{Fml}(V)$ be the set of real formulas $A$ for which $\operatorname{Var}(A) \subseteq V$.

The notation $A(\xi / \varkappa)$ will be used for the formula resulting from replacement of every free occurrence of the variable $x$ in $A$ by $\xi$. If $\xi$ is an apparent variable, this notation is used on the understanding that $\xi$ is free for $\varkappa$ in $A$, that is no free occurrence of $\varkappa$ in $A$ is within the scope of $\forall \xi$.

The individuals $I$ of a stratified model $\mathfrak{A}$ are added as self-designating names to $\mathfrak{L}$ to form $\mathfrak{L}^{I}$. For an $\mathfrak{L}^{I}$-formula $A$, let $I(A)=\{i \in I: i$ occurs in $A\}$, and say that $A$ is defined at the point $t$ of $\mathfrak{A}$ if $I(A) \subseteq D_{t}$. We call $A$ a sentence if it has no free variables, that is $A$ may contain members of $I$ as self-naming constants, but has no occurrences of real variables, and only bound occurrences of apparent variables. Let $\operatorname{Sen}(t)$ be the set of all $\mathfrak{L}^{I}$-sentences that are defined at $t$ in $\mathfrak{A}$.

DEFINITION 2.1. The truth relation $\models$ is defined to hold between theories $t$ of $\mathfrak{A}$ and sentences of $\operatorname{Sen}(t)$ by the clauses

- $t \models R i_{1}, \ldots, i_{n}$ iff $\varphi t\left(R, i_{1}, \ldots, i_{n}\right)$.

- $t=B \wedge C$ iff $t \models B$ and $t \models C$. 
- $t \models \neg B$ iff $(\forall a \geq t)-a \not \models B$.

- $t \models B \rightarrow C$ iff $(\forall u \equiv t)(u \models B$ implies $t u \models C)$.

- $t \models \forall x B$ iff $(\exists t \uparrow)\left(\exists i \in D_{t \uparrow}-D_{t}\right) t \uparrow \models B(i / x)$.

An $\mathfrak{L}^{I}$-sentence $A$ is true in $\mathfrak{A}$, written $\mathfrak{A} \models A$, if $l \models A$ for any $l$ at which $A$ is defined. A real formula $A\left(\mathbf{v}_{1}, \ldots, \mathbf{v}_{n}\right)$ with real variables $\mathbf{v}_{1}, \ldots, \mathbf{v}_{n}$ is true in $\mathfrak{A}$ if for any $i_{1}, \ldots, i_{n} \in I$, and any $l$ at which $A\left(i_{1} / \mathbf{v}_{1}, \ldots, i_{n} / \mathbf{v}_{n}\right)$ is defined, $l \models A\left(i_{1} / \mathbf{v}_{1}, \ldots, i_{n} / \mathbf{v}_{n}\right)$. A real $\mathfrak{L}$-formula is valid in a class of stratified models if it is true in every member of the class.

Using the definition of $\exists x$ as $\neg \forall x \neg$, it is shown in [SQL, Lemma 10] that

$$
t \models \exists x B \text { iff }(\forall a \geq t)(\exists b)\left(\exists i \in D_{b}\right)\left(b \models B(i / x) \text { and } b \downarrow_{a} \leq a\right) .
$$

Axioms and rules for a system BQ are listed in Figure 1. A logic is defined to be any set $\mathrm{L}$ of real formulas that includes all real instances of these axioms and is closed under the rules. If $A \in \mathrm{L}$, we may write $\vdash_{\mathrm{L}} A$ (for " $A$ is an L-theorem"). BQ itself is the smallest logic. A quasi-logic includes all the axioms but need only be closed under the first five rules, and not under generalization.

A model $\mathfrak{A}$ may be said to validate an axiom if all instances of this axiom are true in $\mathfrak{A}$. A rule is valid in $\mathfrak{A}$ if all instances of the rule preserve truth in $\mathfrak{A}$.

We use the term postulate to mean either an axiom or a rule. Figure 2 contains additional standard postulates and corresponding structure conditions [SQL, p. 58]. If $\mathrm{X}$ is any set of these postulates, let BQX be the smallest logic closed under all members of X. RQ is the smallest logic containing all postulates, and EQ the smallest containing all but the last (Assertion) postulate.

It is shown in SQL that BQX is characterized by the class of all stratified models that satisfy all the conditions corresponding to the postulates in X. In other words, a real formula is a theorem of BQX iff it is valid in this class of models. The proof involves constructing a canonical model that satisfies the conditions corresponding to $\mathrm{X}$ while falsifying all non-theorems of BQX. BQ itself is characterized by the class of all models, while RQ is characterized by the class of all models satisfying all the conditions listed in Figure 2. Note that the last condition amounts to commutativity of fusion. ${ }^{4}$

The soundness parts of these characterizations depend on some lemmas, proven by induction on the length of formulas, that hold in any stratified model. These will be stated now. For this we call a sentence $A^{\prime}$ an $i j$-variant of $A$ if it is obtained from $A$ by arbitrarily interchanging some occurrences of $i$ and $j$. For example, the $i j$-variants of $R i j$ are Rii, $R i j, R j i$, and $R j j$.

\section{Lemma 2.2 (Truth Lemmas). For any sentence A:}

(1) Truth Extension: If $A \in \operatorname{Sen}(t)$, then $t \models A$ and $t \leq u$ implies $u \models A$.

(2) Truth Across: If $A^{\prime}$ is an $i j$-variant of $A$, and $t$ is ij-symmetric, then $t \models A$ implies $t \models A^{\prime}$.

(3) Truth Down: If $A \in \operatorname{Sen}(t \downarrow)$, then $t \models A$ iff $t \downarrow \models A$.

(4) Truth Saturation: If $A \in \operatorname{Sen}(t)$ and $(\forall a \geq t) a \models A$, then $t \models A$.

From these results follow certain facts that are used for proving soundness and validity:

4 The excluded-middle postulate $\neg A \vee A$ is included in the definition of BQ in SQL, but the analysis shows that it can be optionally added with its corresponding structure condition. 


\section{Axioms}

$A \rightarrow A$

$A \wedge B \rightarrow A, A \wedge B \rightarrow B$

$(A \rightarrow B) \wedge(A \rightarrow C) \rightarrow(A \rightarrow B \wedge C)$

$(A \rightarrow C) \wedge(B \rightarrow C) \rightarrow(A \vee B \rightarrow C)$

Identity

Conjunction Elimination

Conjunction Introduction

$A \wedge(B \vee C) \rightarrow(A \wedge B) \vee(A \wedge C)$

Disjunction Elimination

$\neg \neg A \rightarrow A$

$\forall x A \rightarrow A(\mathbf{v} / x)$

$\forall x(A \rightarrow B) \rightarrow(A \rightarrow \forall x B)$, with $x$ not free in $A$.

$\wedge \vee$-Distribution

Double-Negation Elimination

Specification

$\forall x(A \vee B) \rightarrow(A \vee \forall x B)$, with $x$ not free in $A$.

Relevant $\forall$-Distribution

Disjunctive $\forall$-Distribution

\section{Rules}

\begin{tabular}{|c|c|}
\hline$\frac{A, B}{A \wedge B}$ & Adjunction \\
\hline$\frac{A, A \rightarrow B}{B}$ & Modus Ponens \\
\hline$\frac{A \rightarrow B}{(B \rightarrow C) \rightarrow(A \rightarrow C)}$ & Suffixing \\
\hline$\frac{B \rightarrow C}{(A \rightarrow B) \rightarrow(A \rightarrow C)}$ & Prefixing \\
\hline$\frac{A \rightarrow \neg B}{B \rightarrow \neg A}$ & Contraposition \\
\hline$\frac{A}{\forall x A(x / \mathbf{v})}$, when $x$ is free for $\mathbf{v}$ in $A$. & Generalisation \\
\hline
\end{tabular}

Fig. 1. Axioms and rules for BQ.

\section{Standard Postulate}

$(A \rightarrow B) \wedge(B \rightarrow C) \rightarrow(A \rightarrow C)$

$(A \rightarrow B) \rightarrow((B \rightarrow C) \rightarrow(A \rightarrow C))$

$(B \rightarrow C) \rightarrow((A \rightarrow B) \rightarrow(A \rightarrow C))$

$(A \rightarrow B) \rightarrow(\neg B \rightarrow \neg A)$

$A \wedge(A \rightarrow B) \rightarrow B$

$(A \rightarrow B) \rightarrow \neg A \vee B$

$(A \rightarrow(B \rightarrow C)) \rightarrow(A \wedge B \rightarrow C)$

$\frac{A}{(A \rightarrow B) \rightarrow B}$

$\neg A \vee A$

$A \rightarrow((A \rightarrow B) \rightarrow B) \quad$ [Assertion]

\section{Condition}

$t(t u) \leq t u$

$t(u v) \leq(u t) v$

$t(u v) \leq(t u) v$

$a \geq t b$ implies $-b \geq t-a$

$t t \leq t$

$a-a \leq a$

(tu) $u \leq t u$

$t l \leq t$

$a \geq l$ implies $a \geq-a$.

$t u \leq u t$

Fig. 2. Standard postulates and their structure conditions.

COROLlary 2.3 (SQL, COROLlary 9).

(1) $a \models \neg B$ iff $-a \not \models B$, where $B \in \operatorname{Sen}(a)$.

(2) $a \models B \vee C$ iff $a \models B$ or $a \models C$, where $B, C \in \operatorname{Sen}(a)$.

(3) $(\forall a \equiv t)(a \models B$ implies ta $\models C)$ implies $t \models(B \rightarrow C)$, where $B, C \in \operatorname{Sen}(t)$.

(4) $l \models(B \rightarrow C)$ iff $(\forall u \equiv l)(u \models B$ implies $u \models C)$ iff $(\forall a \equiv l)(a \models B$ implies $a \models C)$, where $B, C \in \operatorname{Sen}(l)$.

REMARK 2.4 (THE ROLE OF IV(I)(C)). In SQL the condition IV(i)(c) is stated with the stronger conclusion $\exists a^{+} \geq t\left(a^{+} \downarrow=a\right)$, but the proof given of IV(i)(c) in the canonical model only shows the weaker condition $a^{+} \downarrow \leq a$. However this weaker conclusion is all that is needed for the role that IV(i)(c) plays, which is to ensure that the Truth Down Lemma holds for inductive case of $A=\neg B$, as can be readily checked [SQL, p. 41]. 
On the other hand, given IV(i)(c) as stated here, the stronger version can be derived by using IV(i)(b). For if $a^{+} \geq t$ and $a^{+} \downarrow \leq a$, then $-a \leq\left(-a^{+}\right) \downarrow$ (by I(vi) and IV(ii)), so by IV(i)(b) there exists $a^{\prime} \leq-a^{+}$with $a^{\prime} \downarrow=-a$. Putting $a^{*}=-a^{\prime}$, it can then be shown that $a^{*} \geq t$ and $a^{*} \downarrow=a$.

Now the role of IV(i)(b) is to ensure the soundness of the Disjunctive $\forall$-Distribution axiom [SQL, p. 50], while this axiom is used just to show that canonical models satisfy IV(i)(b) [SQL, pp. 54-55]. In other words, this axiom corresponds exactly to IV(i)(b).

The upshot of this discussion is that by stating IV(i)(c) in the weaker form given here, the condition IV(i)(b) can be dropped to obtain a complete semantics for logics that do not include the Disjunctive $\forall$-Distribution axiom.

Further discussion of IV(i)(c) is given in Section 4.

2.1. Conservatively adding $\boldsymbol{\tau}$. The presence of the model elements $l(\alpha)$ makes it natural to enrich the language with a sentential constant $\boldsymbol{\tau}$ having the semantics

$$
t \models \tau \quad \text { iff } \quad l \leq t,
$$

where $l$ here is $l\left(D_{t}\right)$. The cases $A=\tau$ of the Truth Extension, Across and Down lemmas are then readily derived (Truth Down uses II(i)(a,b), III(iii)(a), and IV(iv), showing among other things that $l \downarrow=l$ ). Truth Saturation follows from the additional structural condition

$$
\text { I(viii): If } \forall a \geq t(a \geq l) \text {, then } t \geq l \text {. }
$$

For any logic L, the canonical L-model of [SQL] satisfies this condition. The points in such a model are pairs $t=(\Gamma, V)$ with $V$ a finite set of real variables, and $\Gamma$ a subset of $\operatorname{Fml}(V)$ that is an L-theory. Here $D_{t}=V$, with $\left(\Gamma^{\prime}, V^{\prime}\right) \geq(\Gamma, V)$ iff $\Gamma^{\prime} \supseteq \Gamma$ and $V^{\prime}=V$, while $l(V)=(\operatorname{Fml}(V) \cap \mathrm{L}, V)$. The members of $\mathrm{S}$ are those points for which $\Gamma$ is a prime L-theory in $\operatorname{Fml}(V)$.

Thus if $t \ngtr l$, there is some formula $A \in(\operatorname{Fml}(V) \cap \mathrm{L})-\Gamma$. Lindenbaum’s Lemma then provides a prime L-theory $\Delta$ in $\operatorname{Fml}(V)$ with $\Gamma \subseteq \Delta$ and $A \notin \Delta$. Then $a=(\Delta, V)$ has $a \geq t$ and $a \ngtr l$, verifying I(viii).

To axiomatize logics with $\tau$ under this semantics requires two new postulates. Let $\mathrm{BQX}^{\tau}$ be the logic defined by the axioms and rules for BQ and the postulates from $\mathrm{X}$, all stated for formulas of the language with $\tau$, together with $\tau$ itself as an axiom, and the inference rule

$$
\frac{A}{\tau \rightarrow A}
$$

Completeness for BQX ${ }^{\tau}$ is then shown by handling the $\tau$ case as in [MFE, p. 359].

The following argument, sketched in the Introduction, exemplifies the way we use stratified models to prove conservativity results.

THEOREM 2.5. For any set $X$ of standard postulates, $B Q X^{\tau}$ is a conservative extension of $B Q X$.

Proof. Let $B$ be a $\tau$-free formula that is not a BQX-theorem. We have to show that $B$ is not a BQX ${ }^{\boldsymbol{\tau}}$-theorem. From the above remarks, there is a model $\mathfrak{A}$ for the $\boldsymbol{\tau}$-free language that satisfies I(viii) and whose truth relation $\models$ has $\mathfrak{A} \models \mathrm{BQX}$ but $\mathfrak{A} \not \models B$.

Define a new truth relation $\models^{\prime}$ on $\mathfrak{A}$ for the language including $\boldsymbol{\tau}$, by putting $t \models^{\prime} \boldsymbol{\tau}$ iff $l \leq t$, for all $t \in T$, and otherwise defining $\models^{\prime}$ by exactly the same conditions as for $\models$ given in Definition 2.1. Then an inductive proof shows that any $\boldsymbol{\tau}$-free formula $A$ has $t \models A$ iff $t \models \models^{\prime} A$ for all $t$. Hence $\mathfrak{A} \not \nvdash^{\prime} B$. 
It remains then to show that $\mathfrak{A} \models^{\prime} \mathrm{BQX}^{\boldsymbol{\tau}}$ to obtain our desired result. It is straightforward to check that $\mathfrak{A} \models^{\prime} \boldsymbol{\tau}$, and $\mathfrak{A} \models^{\prime} A$ only if $\mathfrak{A} \models^{\prime} \boldsymbol{\tau} \rightarrow A$. Thus we are left to check that the axioms and rules of BQX are sound in $\mathfrak{A}$ under $\models^{\prime}$. But this follows exactly as in [SQL], since the Truth Lemmas 2.2 all hold with $\models^{\prime}$ in place of $\models$. These lemmas are proven by induction on the length of a formula $A$. When $A$ is $\tau$, the lemmas hold as indicated above. The other cases of $A$ are just as in [SQL], so this completes the argument.

§3. Logics with identity. Assume from now on that the language includes $\tau$, that any logic considered has the postulates for $\tau$ just described, and that all models satisfy I(viii). Next we add a new binary predicate $\approx$. Call a formula $A^{\prime}$ a $\mathbf{v w}$-variant of $A$ if it is obtained from $A$ by arbitrary interchange of some occurrences of the real variables $\mathbf{v}$ and $\mathbf{w}$. Then the axioms for identity that we need are

I1. $A \rightarrow \mathbf{v} \approx \mathbf{v}$

I2. $\mathbf{v} \approx \mathbf{w} \wedge A \rightarrow A^{\prime}$, where $A^{\prime}$ is any $\mathbf{v w - v a r i a n t ~ o f ~} A$

I3. $\mathbf{v} \approx \mathbf{v} \rightarrow(\mathbf{v} \approx \mathbf{v} \rightarrow \mathbf{v} \approx \mathbf{v})$

I4. $\mathbf{v} \approx \mathbf{v} \rightarrow(\mathbf{v} \not \approx \mathbf{v} \rightarrow A)$.

The last two of these can be derived from the others in some logics, as will be explained later (see Remarks 3.6 and 6.9).

Note that by Modus Ponens, $\mathbf{v} \approx \mathbf{v}$ is derivable from I1 (just let $A$ be any theorem), and hence that $\mathbf{v} \not \approx \mathbf{v} \rightarrow A$ is derivable from I4.

The definition of a structure $\mathfrak{A}$ for this language remains as before, on the understanding that the valuation relation $\varphi t\left(R, i_{1}, \ldots, i_{n}\right)$ now includes the case that $R$ is $\approx$ and $n=2$. For each $t \in T$ we define a binary relation $\approx_{t}$ on $D_{t}$ by putting $i \approx_{t} j$ iff $\varphi t(\approx, i, j)$. A stratified identity model is a structure that satisfies the conditions I-V as before, and also the following:

\section{Behavior of $\approx$}

(i) $i \approx_{t} j$ iff $t={ }^{i j} \vec{t}$.

The definition of the truth relation $\models$ remains as in Definition 2.1. In particular, for an identity sentence $i \approx j$ we get that

$$
t \models i \approx j \quad \text { iff } \quad \varphi t(\approx, i, j) \quad \text { iff } i \approx_{t} j .
$$

Since the new identity predicate $\approx$ behaves like other predicates in satisfying I(vii), III(iv), and V(ix), the proofs of the Truth Lemmas 2.2 hold for this language with identity, just as in SQL.

\section{LEMMA 3.1.}

(1) $t \leq u$ implies $\approx_{t} \subseteq \approx_{u}$.

(2) $i \approx_{t} i$, for all $i \in D_{t}$.

(3) If $t={ }^{i j} \vec{t}$ and $t \leq u$, then $u={ }^{i j} \vec{u}$.

\section{Proof.}

(1) This is really a manifestation of Truth Extension for identities, but we give the details ab initio. Let $t \leq u$. If $i \approx_{t} j$, then by I(vii), $(\forall a \geq t) a \models i \approx_{a} j$, hence $(\forall a \geq u) a \models i \approx_{a} j$ by transitivity, so $i \approx_{u} j$ by I(vii) again. 
(2) Given $i \in D_{t}$, by II(i) there exists a $\beta \in \mathfrak{D}$ with $D_{t} \subset \beta$, so there is some $j \in$ $\beta-D_{t}$, hence $j \neq i$. Let $t \uparrow$ be $t \uparrow^{\beta}$ and $\rightarrow$ be ${ }^{i j} \overrightarrow{\text {. Put }} u=\overrightarrow{(t \uparrow)}$. Then $u=\vec{u}$ by V(i,iii), so $i \approx_{u} j$ by the new VI(i). But then $i \approx_{u} i$ by V(ix) (remember $i \approx_{u} j$ means $\left.\varphi u(\approx, i, j)\right)$. Now $i \in D_{u \downarrow}=D_{t}$, so then $i \approx_{u \downarrow} i$ by III(iv). But

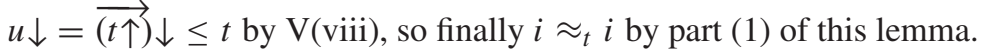

(3) Let $t={ }^{i j} \vec{t}$. Then $i \approx_{t} j$ by VI(i), so if $t \leq u$ then $i \approx_{u} j$ by (1), hence $u={ }^{i j} \vec{u}$ by VI(i).

\section{COROLLARY 3.2. The identity axioms II-I4 are valid in stratified identity models.}

Proof. From Lemma 3.1(2) it follows that $t \models i \approx i$ for any $i \in D_{t}$, so a self-identity sentence can never be falsified at any point in such a model. Consequently, no sentence of the form $A \rightarrow i \approx i$ can be falsified, ensuring that axiom I1 is valid. In particular, $i \approx i \rightarrow i \approx i$ can never be falsified, ensuring the validity of I3.

For I2, if $A^{\prime}$ is an $i j$-variant of sentence $A \in \operatorname{Sen}(t)$, and $t \models i \approx j \wedge A$, then $i \approx_{t} j$ and $t \models A$, so $t={ }^{i j} \vec{t}$ by VI(i), hence $t \models A^{\prime}$ by the Truth Across Lemma.

For I4 it suffices to show $t \models i \not z i \rightarrow A$ for any $A \in \operatorname{Sen}(t)$. But if not, there exists $u \equiv t$ with $u \models i \not \approx i$ while $t u \not \models A$. Then by Truth Saturation, there exists $a \geq t u$ with $a \not \models A$. Hence by I(iii), there is some $b \in S$ with $b \geq u$ (and $a \geq t b$ ). Since $u \models i \not \approx i$, Truth Extension and the semantics of $\neg$ then imply $-b \not \models i \approx i$, which contradicts what we just proved about self-identities being unfalsifiable. This shows that I4 is valid.

We turn now to completeness theorems for logics with identity. If $\mathrm{L}$ is a quasi-logic, we write $\Delta \vdash_{\mathrm{L}} B$, and say that $B$ is $L$-deducible from the set of formulas $\Delta$, if there exists an L-deduction of $B$ from $\Delta$, that is a finite sequence of formulas $A_{0}, \ldots, A_{n}$ such that $A_{n}=B$ and for all $m \leq n$, either $A_{m} \in \Delta$ or $(\exists p, q<m)\left(A_{m}=A_{p} \wedge A_{q}\right)$ or $(\exists p<m)\left(\vdash_{L} A_{p} \rightarrow A_{m}\right)$. This is equivalent to requiring that there exist members $B_{0}, \ldots, B_{k}$ of $\Delta$ such that $\vdash_{\llcorner} B_{0} \wedge \cdots \wedge B_{k} \rightarrow B$. We assume properties of this deducibility relation shown in [MFE, SQL], including the Deduction Theorem: $A \vdash_{L} B$ iff $\vdash_{L} A \rightarrow B$. Note that if $\Delta$ is closed under conjunction, then $\Delta \vdash_{L} B$ iff there exists $A \in \Delta$ with $A \vdash_{L} B$.

$\Delta$ is called an L-theory if it is closed under L-deduction, that is $\Delta \vdash_{L} B$ implies $B \in \Delta$; L-prime if $\Delta \vdash_{L} A \vee B$ implies $\Delta \vdash_{L} A$ or $\Delta \vdash_{L} B$; proper if there is some formula not in $\Delta$; and L-saturated if it is an L-prime and proper L-theory. In general, $\left\{B: \Delta \vdash_{L} B\right\}$ is the least L-theory including the set $\Delta$, and if $\Delta$ is closed under conjunction, then this is just $\{B: \exists A \in \Delta(A \vdash B)\}$.

Lindenbaum's Lemma. If $\Gamma$ is a set of formulas closed under disjunction, and $\Delta$ is an $L$-theory that does not intersect $\Gamma$, then $\Delta$ has an L-prime extension $\Delta^{\prime}$ that does not intersect $\Gamma$. If $\Gamma \neq \emptyset$, then $\Delta^{\prime}$ is L-saturated.

The first sentence of this result is proved as in [MFE, pp. 352-353], and the second follows immediately, since $\Gamma \neq \emptyset$ implies $\Delta^{\prime}$ is proper. As a special case we have:

If $\Delta$ is an L-theory and $A \notin \Delta$, then $\Delta$ has a proper L-prime extension not containing $A$.

Recall that $\operatorname{Fml}(V)$ is the set of formulas $A$ whose set $\operatorname{Var}(A)$ of real variables is included in $V$. The above notions and results relativize to $\operatorname{Fml}(V)$. Thus if $\Delta \subseteq \operatorname{Fml}(V)$, then $\Delta$ is an L-theory in $V$ if $B \in \Delta$ whenever $\Delta \vdash_{\mathrm{L}} B$ and $B \in \operatorname{Fml}(V)$; and is L-prime in $V$ if 
$\Delta \vdash_{L} A \vee B$ and $A \vee B \in \operatorname{Fml}(V)$ implies $\Delta \vdash_{L} A$ or $\Delta \vdash_{L} B . \Delta$ is L-saturated in $V$ if it is an L-theory in $V$ that is L-prime in $V$ and a proper subset of $\operatorname{Fml}(V)$.

Now let $\mathrm{L}$ be a logic that includes the identity axioms. For distinct real variables $\mathbf{v}, \mathbf{w}$, let $\mathrm{L}^{\mathrm{vw}}$ be the smallest quasi-logic to include $\mathrm{L}$, all formulas of the type $A \rightarrow A^{\prime}$ where $A^{\prime}$ is a vw-variant of $A$, and all formulas of the type $A \rightarrow \mathbf{v} \approx \mathbf{w}$. The inclusion of the latter type of formula is a strengthening of the definition of $\mathrm{L}^{\mathbf{v w}}$ from [SQL], so we need to reprove the following result, which is [SQL, Lemma 15].

LEMma 3.3. For distinct real variables $\mathbf{v}, \mathbf{w}$, and formulas $A$ and $B$, the following are equivalent.

(1) $A \vdash^{\mathrm{vw}} B$.

(2) $\vdash_{\mathrm{L}^{\mathrm{vw}}} A \rightarrow B$.

(3) $\vdash_{\mathrm{L}} A(\mathbf{v} / \mathbf{w}) \rightarrow B(\mathbf{v} / \mathbf{w})$.

Proof. (1) implies (2): by the Deduction Theorem. (2) implies (3): an induction on proofs is used to show that $\vdash_{\mathrm{L}^{\mathrm{vw}}} C$ implies $\vdash_{\mathrm{L}} C(\mathbf{v} / \mathbf{w})$ for any formula $C$. The one new case is when $C$ is $A \rightarrow \mathbf{v} \approx \mathbf{w}$. But then $C(\mathbf{v} / \mathbf{w})$ is $A(\mathbf{v} / \mathbf{w}) \rightarrow \mathbf{v} \approx \mathbf{v}$, an instance of the identity axiom I1 and hence an L-theorem.

(3) implies (1): if $\vdash_{\mathrm{L}} A(\mathbf{v} / \mathbf{w}) \rightarrow B(\mathbf{v} / \mathbf{w})$, then the sequence $A, A(\mathbf{v} / \mathbf{w}), B(\mathbf{v} / \mathbf{w}), B$ is an $\mathrm{L}^{\mathbf{v w}}$-deduction of $B$ from $A$.

An L- $V$-theory is defined to be a pair $t=(\Delta, V)$ with $\Delta$ a nonempty L-theory in $V$. We write $\operatorname{Thm}(t)$ for $\Delta$ and $\operatorname{Var}(t)$ for $V . t$ is called L- $V$-saturated if $\operatorname{Thm}(t)$ is L-saturated (i.e. L-prime and proper) in $V$. For a set $U$ of real variables, let $t \mid U=(\Gamma, U)$, where $\Gamma=\left\{A: \operatorname{Var}(A) \subseteq U\right.$ and $\left.\operatorname{Thm}(t) \vdash_{\mathrm{L}} A\right\}$. Then $t \mid U$ is an L- $U$-theory. When $V \subseteq U$, $t \mid U$ is the expansion of $t$ to $U$, and is the least L-theory in $U$ that includes $\operatorname{Thm}(t)$. When $U \subseteq V, t \mid U$ is the contraction of $t$ to $U$, in which case $\operatorname{Thm}(t \mid U)=\{A \in \operatorname{Thm}(t)$ : $\operatorname{Var}(A) \subseteq U\}$.

The canonical model $\mathfrak{A}_{\mathrm{L}}$ for the logic $\mathrm{L}$ has the following structure [SQL, p. 53]:

(i) $T=\{t: T$ is an $\mathrm{L}-V$-theory for a finite nonempty set $V\}$.

(ii) $S=\{t \in T: t$ is $\mathrm{L}-V$-saturated $\}$.

(iii) $D=\{(t, \operatorname{Var}(t)): t \in T\}$.

Hence $\mathfrak{D}=\{\operatorname{Var}(t)): t \in T\}=\{V: V$ is a finite nonempty set of real variables $\}$.

(iv) $l=\{(V, t): t \in T, \operatorname{Var}(t)=V$, and $\operatorname{Thm}(t)=\mathrm{L} \cap \operatorname{Fml}(V)\}$.

(v) $\cdot=\{(t, u, v): t, u, v \in T, \operatorname{Var}(t)=\operatorname{Var}(u)=\operatorname{Var}(v)$, and

$\operatorname{Thm}(v)=\{B:(\exists A \in \operatorname{Thm}(u)) A \rightarrow B \in \operatorname{Thm}(t)\}\}$.

(vi) $-=\left\{(a, b) \in S^{2}: \operatorname{Var}(b)=\operatorname{Var}(a)\right.$ and $\operatorname{Thm}(b)=\{B: \neg B \notin \operatorname{Thm}(a)\}$.

(vii) $\geq=\left\{(t, u) \in T^{2}: \operatorname{Var}(u)=\operatorname{Var}(t)\right.$ and $\left.\operatorname{Thm}(u) \subseteq \operatorname{Thm}(t)\right\}$.

(viii) $\uparrow=\{(t, \alpha, u): t, u \in T, \operatorname{Var}(t) \subseteq \alpha \in \mathfrak{D}$, and $u$ is the expansion of $t$ to $\alpha\}$.

(ix) $\downarrow=\{(t, \alpha, u): t, u \in T, \emptyset \neq \alpha \subseteq \operatorname{Var}(t)$, and $u$ is the contraction of $t$ to $\alpha\}$.

(x) $\rightarrow=\{(t, \mathbf{v}, \mathbf{w}, u): t, u \in T, \mathbf{v}, \mathbf{w} \in \operatorname{Var}(t), \mathbf{v} \neq \mathbf{w}, \operatorname{Var}(u)=\operatorname{Var}(t)$, and

$\operatorname{Thm}(u)$ is the least $\mathrm{L}^{\mathbf{v w}}$-theory including $\left.\operatorname{Thm}(t)\right\}$.

Thus $\operatorname{Thm}(t) \subseteq \operatorname{Thm}\left({ }^{\mathbf{v w}} \vec{t}\right)=\left\{B: \exists A \in \operatorname{Thm}(t)\left(A \vdash^{\mathbf{v w}} B\right)\right\}$.

(xi) $\varphi t\left(R, \mathbf{v}_{1}, \ldots, \mathbf{v}_{n}\right)$ iff $R \mathbf{v}_{1} \ldots \mathbf{v}_{n} \in \operatorname{Thm}(t)$.

LEMMA 3.4. $\mathfrak{A}_{\mathrm{L}}$ is a stratified identity model. 
Proof. The proof that $\mathfrak{A}_{\mathrm{L}}$ satisfies the conditions of groups I-V is essentially as in [MEF, $\mathrm{SQL}]$, except that those papers did not require, as we do here, that $\operatorname{Thm}(t)$ be nonempty, and that it be a proper subset of $\operatorname{Fml}(\operatorname{Var}(t))$ when $t$ is saturated. So we need to check those points where these requirements need to be verified.

First we need to verify that the operations on $\mathfrak{A}_{\mathrm{L}}$ are well-defined. Note that if $t \in T$, then there exists some $A \in \operatorname{Thm}(t)$, so by axiom I1 we get $\mathbf{v} \approx \mathbf{v} \in \operatorname{Thm}(t)$ for each $\mathbf{v} \in \operatorname{Var}(t)$. Hence axiom I3 yields $(\mathbf{v} \approx \mathbf{v} \rightarrow \mathbf{v} \approx \mathbf{v}) \in \operatorname{Thm}(t)$. Thus if $u \in T$ has $\operatorname{Var}(u)=\operatorname{Var}(t)$, then taking any $\mathbf{v} \in \operatorname{Var}(t)$ we have $(\mathbf{v} \approx \mathbf{v} \rightarrow \mathbf{v} \approx \mathbf{v}) \in \operatorname{Thm}(t)$ and $\mathbf{v} \approx \mathbf{v} \in \operatorname{Thm}(u)$, so $\mathbf{v} \approx \mathbf{v} \in \operatorname{Thm}(t \cdot u)$, ensuring $\operatorname{Thm}(t \cdot u) \neq \emptyset$ as required.

Now let $a \in S$. We need to check that the prime theory $\operatorname{Thm}(-a)=\{B: \neg B \notin$ $\operatorname{Thm}(a)\}$ is nonempty and proper, hence saturated. Now $\operatorname{Thm}(a)$ is proper, so there is some $A \in \operatorname{Fml}(\operatorname{Var}(a))-\operatorname{Thm}(a)$. But the Double-Negation Elimination axiom yields $\neg \neg A \notin \operatorname{Thm}(a)$, so $\neg A \in \operatorname{Thm}(-a)$. Also there exists a $B \in \operatorname{Thm}(a)$, and $\vdash_{\mathrm{L}} B \rightarrow \neg \neg B$, so $\neg \neg B \in \operatorname{Thm}(a)$, and so $\neg B \notin \operatorname{Thm}(-a)$.

For the up operator, if $t \in T$ and $\operatorname{Var}(t) \subseteq \alpha$, then $\operatorname{Thm}(t) \subseteq \operatorname{Thm}\left(t \uparrow^{\alpha}\right)$, so $\operatorname{Thm}\left(t \uparrow^{\alpha}\right)$ is nonempty because $\operatorname{Thm}(t)$ is. Similarly for the across operator, as $\operatorname{Thm}(t) \subseteq \operatorname{Thm}(\vec{t})$.

For the down operator, if $\mathbf{v} \in \alpha \subseteq \operatorname{Var}(t)$, then $\mathbf{v} \approx \mathbf{v} \in \operatorname{Thm}\left(t \downarrow_{\alpha}\right)$. Actually, it is not necessary to use properties of identity to show $\operatorname{Thm}\left(t \downarrow_{\alpha}\right) \neq \varnothing$. For if $B \in \operatorname{Thm}(t)$, and $\mathbf{v}_{1}, \ldots, \mathbf{v}_{n}$ are all the members of $\operatorname{Var}(t)-\alpha$, then $\vdash_{\mathrm{L}} B \rightarrow \exists x_{1} \ldots \exists x_{n} B\left(x_{1} / \mathbf{v}_{1}, \ldots, x_{n} / \mathbf{v}_{n}\right)$ where $x_{1} \ldots x_{n}$ are new variables (cf. Lemma 4.1(1)), so $\exists x_{1} \ldots \exists x_{n} B\left(x_{1} / \mathbf{v}_{1}, \ldots, x_{n} / \mathbf{v}_{n}\right)$ belongs to $\operatorname{Thm}(t) \cap \operatorname{Fml}(\alpha)$, hence to $\operatorname{Thm}\left(t \downarrow_{\alpha}\right)$.

The points from conditions $\mathrm{I}-\mathrm{V}$ that need checking are as follows.

I(iii): Let $a \geq t u$, where $a, t, u$ are $\mathrm{L}, V$-theories. To show $\exists b \geq t(a \geq b u)$, let

$$
\Gamma=\{A \in \operatorname{Fml}(V): \exists B, C(A \vdash B \rightarrow C \& B \in \operatorname{Thm}(u) \& C \notin \operatorname{Thm}(a)\} .
$$

Then $\Gamma$ is closed under disjunction [MFE, p. 354], and is disjoint from $\operatorname{Thm}(t)$. Hence by Lindenbaum's Lemma there exists a $\Delta$ extending $\operatorname{Thm}(t)$ that is L-prime in $V$ and disjoint from $\Gamma$. But $\operatorname{Thm}(a)$ is proper and $\operatorname{Thm}(u)$ is nonempty, so taking any $C \in \operatorname{Fml}(V)-$ $\operatorname{Thm}(a)$ and $B \in \operatorname{Thm}(u)$ gives $B \rightarrow C \in \Gamma$. Hence $\Gamma \neq \emptyset$, so $\Delta$ is proper and nonempty, and $b=(\Delta, V)$ is saturated, hence in $S$, with $a \geq b u$.

Also we want that $\exists b \geq u(a \geq t b)$. Here we use the identity axiom I4. Let

$$
\Gamma=\{A \in \operatorname{Fml}(V): \exists B \notin \operatorname{Thm}(a)(A \rightarrow B \in \operatorname{Thm}(t))\} .
$$

Again $\Gamma$ is closed under disjunction and disjoint from $\operatorname{Thm}(t)$. Take any $B \in \operatorname{Fml}(V)-$ $\operatorname{Thm}(a)$ and $\mathbf{v} \in V$. Then $\mathbf{v} \approx \mathbf{v} \in \operatorname{Thm}(t)$, so by I4, $(\mathbf{v} \neq \mathbf{v} \rightarrow B) \in \operatorname{Thm}(t)$, giving $\mathbf{v} \neq \mathbf{v} \in \Gamma$. So $\Gamma \neq \emptyset$, and again we obtain a $b=(\Delta, V) \in S$ as desired with $\Delta$ a prime extension of $\operatorname{Thm}(t)$ disjoint from $\Gamma$.

I(vii): If $R \mathbf{v}_{1} \ldots \mathbf{v}_{n} \notin \operatorname{Thm}(t)$, then there is a prime extension $\Delta$ of $\operatorname{Thm}(t)$ with $R \mathbf{v}_{1} \ldots \mathbf{v}_{n} \notin \Delta$. Then $\Delta$ is proper, so $a=(\Delta, \operatorname{Var}(t))$ has $a \in S$ with $a \geq t$ but not $\varphi a\left(R, \mathbf{v}_{1}, \ldots, \mathbf{v}_{n}\right)$.

IV(i)(a): We want $a \downarrow$ to be in $S$ whenever $a \in S$. Now Thm $(a \downarrow)$ will be a prime L-theory in $\operatorname{Var}(a \downarrow)$ when $\operatorname{Thm}(a)$ is a a prime L-theory in $\operatorname{Var}(a)$, and nonempty when $\operatorname{Thm}(a)$ is nonempty as shown above. The new point is that $\operatorname{Thm}(a \downarrow)$ is proper. But there exists $A \notin \operatorname{Thm}(a)$, and if $\operatorname{Var}(a)-\operatorname{Var}(a \downarrow)=\left\{\mathbf{v}_{1}, \ldots, \mathbf{v}_{n}\right\}$ then taking fresh variables $x_{1}, \ldots, x_{n}$, the formula $\forall x_{1} \ldots \forall x_{n} A\left(x_{1} / \mathbf{v}_{1}, \ldots, x_{n} / \mathbf{v}_{n}\right)$ is not in $\operatorname{Thm}(a)$ by the specification axiom, but is in $\operatorname{Fml}(\operatorname{Var}(a \downarrow))$, so is not in $\operatorname{Thm}(a \downarrow)$.

IV(i)(b): Let $a \leq b \downarrow$. We need an $a^{+} \leq b$ with $a^{+} \downarrow=a$. This is constructed in [SQL] in the form $A^{+}=\left(\Delta^{+}, \operatorname{Var}(b)\right)$, where $\Delta^{+}$is a prime theory in $\operatorname{Var}(b)$ that extends the 
expansion of $a$ to $\operatorname{Var}(b)$ and is disjoint from $\Sigma^{\prime}$, where $\Sigma^{\prime}$ in turn is the closure under disjunction of

$$
[\operatorname{Fml}(\operatorname{Var}(a))-\operatorname{Thm}(a)] \cup[\operatorname{Fml}(\operatorname{Var}(b))-\operatorname{Thm}(b)] .
$$

But $[\operatorname{Fml}(\operatorname{Var}(a))-\operatorname{Thm}(a)]$ is nonempty, since $\operatorname{Thm}(a)$ is proper, so $\Sigma^{\prime}$ is nonempty, making $\Delta^{+}$proper as required.

IV(i)(c): Let $a \geq t \downarrow$. To construct $a^{+}=\left(\Delta^{+}, \operatorname{Var}(t)\right)$ with $a^{+} \geq t$ and $a^{+} \downarrow \leq a$, let $\overline{\operatorname{Thm}}(a)=\operatorname{Fml}(\operatorname{Var}(a))-\operatorname{Thm}(a)$. Then $\operatorname{Thm}(t)$ is disjoint from $\overline{\operatorname{Thm}}(a)$, which is closed under disjunction as $\operatorname{Thm}(a)$ is prime in $\operatorname{Var}(a)$. Hence $\operatorname{Thm}(t)$ extends to the desired L-theory $\Delta^{+}$that is L-prime in $\operatorname{Var}(t)$ and disjoint from $\overline{\operatorname{Thm}}(a)$. But $\overline{\operatorname{Thm}}(a) \neq \varnothing$, as $\operatorname{Thm}(a)$ is proper, hence $\Delta^{+}$is proper, and $a^{+} \in S$ as required.

V(iv): If $t$ is vw-symmetric and $t \leq a \in S$, then [SQL, p. 56] constructs a vw-symmetric object $a^{\prime}$ such that $t \leq a^{\prime} \leq a$ and $\operatorname{Thm}\left(a^{\prime}\right)$ is an $\mathrm{L}^{\mathbf{v w}}$-prime $\mathrm{L}^{\mathbf{v w}}$-theory in $\operatorname{Var}(t)$. But then $\operatorname{Thm}\left(a^{\prime}\right)$ is proper in $\operatorname{Var}(t)$, as $\operatorname{Thm}\left(a^{\prime}\right) \subseteq \operatorname{Thm}(a)$ and $\operatorname{Thm}(a)$ is proper, so $a^{\prime} \in S$ as required.

That completes the review of properties I-V. For VI(i), suppose that $\mathbf{v} \approx_{t} \mathbf{w}$, that is $\varphi t(\approx, \mathbf{v}, \mathbf{w})$, which means in $\mathfrak{A}_{\mathrm{L}}$ that $\mathbf{v} \approx \mathbf{w} \in \operatorname{Thm}(t)$. We want to show $t=\vec{t}$, where $\rightarrow$ is $\mathbf{v w} \rightarrow$. The argument is similar to Mares (1992, Lemma 6.2). First, from axiom I2, Thm $(t)$ is closed under vw-variants, that is if $A^{\prime}$ is a vw-variant of $A \in \operatorname{Thm}(t)$, then $\mathbf{v} \approx \mathbf{w} \wedge A \in$ $\operatorname{Thm}(t)$, and so from $\mathrm{I} 2, A^{\prime} \in \operatorname{Thm}(t)$. Now if $B \in \operatorname{Thm}(\vec{t})$, there exists $A \in \operatorname{Thm}(t)$ with $A \vdash^{\mathbf{v}^{\mathbf{w}}} B$. Then by Lemma 3.3, $\vdash_{\mathrm{L}} A(\mathbf{v} / \mathbf{w}) \rightarrow B(\mathbf{v} / \mathbf{w})$. But $A(\mathbf{v} / \mathbf{w}) \in \operatorname{Thm}(t)$ by closure under vw-inviariants, hence $B(\mathbf{v} / \mathbf{w}) \in \operatorname{Thm}(t)$ as $\operatorname{Thm}(t)$ is an L-theory, and so finally $B \in \operatorname{Thm}(t)$ as $B$ is a vw-variant of $B(\mathbf{v} / \mathbf{w})$. Hence $\operatorname{Thm}(\vec{t})=\operatorname{Thm}(t)$ as required.

Conversely, suppose $t=\vec{t}$. Take $A \in \operatorname{Thm}(\vec{t})$. Now $A \vdash_{\mathrm{L}^{\mathrm{vw}}} \mathbf{v} \approx \mathbf{w}$, by definition of $\mathrm{L}^{\mathbf{v w}}$, and $\operatorname{Thm}(\vec{t})$ is an $\mathrm{L}^{\mathbf{v w}}$-theory, so $\mathbf{v} \approx \mathbf{w} \in \operatorname{Thm}(\vec{t})=\operatorname{Thm}(t)$, hence $\mathbf{v} \approx_{t} \mathbf{w}$.

Let $\mathrm{BQX} \tau \approx$ be the logic in the language with $\tau$ and $\approx$ that is defined by the axioms and rules for $\mathrm{BQ}$, the postulates from $\mathrm{X}$, the postulates for $\tau$, and the identity axioms I1-I4.

THEOREM 3.5. If $X$ is any set of the standard postulates of Figure 2, then the logic $B Q X \tau \approx$ is characterized by the class of all stratified identity models satisfying the structure conditions corresponding to the members of $X$.

Proof. Soundness has already been discussed. For completeness, let $\mathrm{L}=\mathrm{BQX} \boldsymbol{\tau} \approx$, and suppose $B$ is a real formula with $\nvdash_{\mathrm{L}} B$. The stratified identity model $\mathfrak{A}_{\mathrm{L}}$ satisfies the structure conditions corresponding to $\mathrm{X}$, hence validates $\mathrm{L}$, so it suffices to show $\mathfrak{A}_{\mathrm{L}} \not \forall B$.

Now the real variables can be regarded as self-designating names for the individuals in $\mathfrak{A}_{\mathrm{L}}$, and so any real formula $A$ can be subject to the truth relation $t \models A$ in $\mathfrak{A}_{\mathrm{L}}$ when $\operatorname{Var}(A) \subseteq \operatorname{Var}(t)$. The Canonical Truth Lemma states that in $\mathfrak{A}_{\mathrm{L}}$,

$$
t \models A \quad \text { iff } \quad A \in \operatorname{Thm}(t) .
$$

This is proven as in [SQL, Lemma 17]. The only new case here is when $A$ is an atomic identity, but this holds just as for any other atomic formula, and indeed was indicated above: $t \models \mathbf{v} \approx \mathbf{w}$ iff $\varphi t(\approx, \mathbf{v}, \mathbf{w})$ iff $\mathbf{v} \approx \mathbf{w} \in \operatorname{Thm}(t)$. So taking any $V \in \mathfrak{D}$ with $\operatorname{Var}(B) \subseteq V$ we get $B \notin l(V)=\mathrm{L} \cap \operatorname{Fml}(V)$, and hence $l(V) \not \forall B$ in $\mathfrak{A}_{\mathrm{L}}$.

REMARK 3.6 (The Role of AXIOM I4). I4 has been used only to prove that the canonical model $\mathfrak{A}_{\mathrm{L}}$ satisfies the second part of condition $\mathrm{I}(\mathrm{iii})$, that is that $a \geq t u$ implies $\exists b \geq u(a \geq t b)$. The first part of I(iii), that $a \geq t u$ implies $\exists b \geq t(a \geq b u)$, was shown without any use of identity axioms. But if the fusion operation is commutative, that 
is $t u=u t$, then the second part of I(iii) follows from the first. Thus for logics containing the Assertion axiom $A \rightarrow((A \rightarrow B) \rightarrow B)$ (see last line of Figure 2), we do not need I4, and can carry through the completeness proof for identity systems by using only I1-I3.

It must follow that in such systems, I4 is derivable from the other axioms. Indeed, from Assertion we can derive the Permutation rule

$$
\frac{A \rightarrow(B \rightarrow C)}{B \rightarrow(A \rightarrow C)}
$$

But the schema $\mathbf{v} \not \approx \mathbf{v} \rightarrow B$ is derivable from I1 using Double-Negation Elimination. In particular we can derive $\mathbf{v} \not \approx \mathbf{v} \rightarrow(\mathbf{v} \approx \mathbf{v} \rightarrow A)$, from which I4 follows by the Permutation rule.

For logics in which I3 is derivable, and further information about the role of I4, see Remark 6.9.

§4. The structural role of conjunctive existential distribution. Figure 3 lists postulates involving the existential quantifier that are derivable in $\mathrm{BQ}$. Their derivation depends on Double-Negation Elimination and the definition of $\exists x$ as $\neg \forall x \neg$. But in Section 6 we will consider logics in which Double-Negation Elimination is not present, and $\exists$ is primitive. A semantics for $\exists$ will be given that validates the Existence axiom and the $\exists$-Elimination rule. Validity of Conjunctive $\exists$-Distribution will require the new structural condition

$$
\text { IV(i)(d): } u \geq t \downarrow \text { implies } \exists u^{+} \geq t\left(u^{+} \downarrow=u\right) .
$$

It is noteworthy that in the presence of $\operatorname{IV}(\mathrm{i})(\mathrm{d})$, the condition

$$
\text { IV(i)(c): } a \geq t \downarrow \text { implies } \exists a^{+} \geq t\left(a^{+} \downarrow \leq a\right)
$$

implies the stronger

$$
\text { IV(i)(c)': } a \geq t \downarrow \text { implies } \exists a^{+} \geq t\left(a^{+} \downarrow=a\right)
$$

that was discussed earlier in Remark 2.4. For if $a \geq t \downarrow$, then from IV(i)(d) we get some $u \geq t$ with $u \downarrow=a$, and then as $a \geq u \downarrow$, from IV(i)(c) we get some $a^{+} \geq u$ with $a^{+} \downarrow \leq a$. But now $a^{+} \geq t$, and $a^{+} \downarrow \geq u \downarrow=a$, hence $a^{+} \downarrow=a$.

Canonical models for BQ and related logics can be shown to satisfy IV(i)(d). We now give a proof of this whose only quantifier-related prerequisites are the postulates of Figure 3. Hence this proof is available for logics having these postulates with $\exists$ primitive.

LEMMA 4.1. Let $\mathrm{L}$ be a logic having the $\exists$-postulates of Figure 3. Suppose that $x_{1}, \ldots, x_{n}$ are distinct and do not occur in $A$ or $B$, and that $\mathbf{v}_{1}, \ldots, \mathbf{v}_{n}$ are distinct and do not occur in A. Then

(1) $\vdash_{\mathrm{L}} B \rightarrow \exists x_{n} \ldots \exists x_{1} B\left(x_{1} / \mathbf{v}_{1}, \ldots, x_{n} / \mathbf{v}_{n}\right)$.

(2) $\vdash_{\mathrm{L}} A \wedge \exists x_{n} \ldots \exists x_{1} B\left(x_{1} / \mathbf{v}_{1}, \ldots, x_{n} / \mathbf{v}_{n}\right) \rightarrow \exists x_{n} \ldots \exists x_{1}\left(A \wedge B\left(x_{1} / \mathbf{v}_{1}, \ldots, x_{n} / \mathbf{v}_{n}\right)\right)$.

Proof. We use the principle that if $x$ is not in $C$, then $\vdash_{\mathrm{L}} C \rightarrow \exists x C(x / \mathbf{v})$, by the Existence axiom as $[C(x / \mathbf{v})](\mathbf{v} / x)$ is just $C$. The proofs are by induction on $n$.

$$
\begin{array}{ll}
A(\mathbf{v} / x) \rightarrow \exists x A & \text { Existence } \\
\frac{A \rightarrow B}{\exists x A(x / \mathbf{v}) \rightarrow B} \text { when } \mathbf{v} \text { does not occur in } B & \text { ヨ-Elimination } \\
A \wedge \exists x B \rightarrow \exists x(A \wedge B), \text { with } x \text { not free in } A & \text { Conjunctive } \exists \text {-Distribution }
\end{array}
$$

Fig. 3. Postulates for $\exists$. 
(1) Let $B_{n}=\exists x_{n} \ldots \exists x_{1} B\left(x_{1} / \mathbf{v}_{1}, \ldots, x_{n} / \mathbf{v}_{n}\right)$. As $x_{n+1}$ is not in $B_{n}, \vdash_{L} B_{n} \rightarrow$ $\exists x_{n+1} B_{n}\left(x_{n+1} / \mathbf{v}_{n+1}\right)$ by the above principle, that is $\vdash_{L} B_{n} \rightarrow B_{n+1}$. Hence if $\vdash_{L} B \rightarrow B_{n}$, then $\vdash_{L} B \rightarrow B_{n+1}$. But $\vdash_{L} B \rightarrow B_{1}=\exists x_{1} B\left(x_{1} / \mathbf{v}_{1}\right)$ as $x_{1}$ is not in $B$, so we get $\vdash_{L} B \rightarrow B_{n}$ for all $n$ by induction on $n$.

(2) Let

$$
\begin{aligned}
& L_{n}=A \wedge \exists x_{n} \ldots \exists x_{1} B\left(x_{1} / \mathbf{v}_{1}, \ldots, x_{n} / \mathbf{v}_{n}\right) \\
& R_{n}=\exists x_{n} \ldots \exists x_{1}\left(A \wedge B\left(x_{1} / \mathbf{v}_{1}, \ldots, x_{n} / \mathbf{v}_{n}\right)\right),
\end{aligned}
$$

the left and right sides of (2). Then $L_{1} \rightarrow R_{1}$ is a Conjunctive $\exists$-Distribution axiom. Assume inductively that $\vdash_{\mathrm{L}} L_{n} \rightarrow R_{n}$. Now $x_{n+1}$ does not occur in $R_{n}$, so $\vdash_{\mathrm{L}}$ $R_{n} \rightarrow \exists x_{n+1} R_{n}\left(x_{n+1} / \mathbf{v}_{n+1}\right)$. Since $\mathbf{v}_{n+1}$ is not in $A, \exists x_{n+1} R_{n}\left(x_{n+1} / \mathbf{v}_{n+1}\right)$ is just $R_{n+1}$. It follows that $\vdash_{\mathrm{L}} L_{n} \rightarrow R_{n+1}$. But $\mathbf{v}_{n+1}$ is not in $R_{n+1}$, so the $\exists$-Elimination rule then gives $\vdash_{\llcorner} \exists x_{n+1} L_{n}\left(x_{n+1} / \mathbf{v}_{n+1}\right) \rightarrow R_{n+1}$. Now

$\exists x_{n+1} L_{n}\left(x_{n+1} / \mathbf{v}_{n+1}\right)=\exists x_{n+1}\left(A \wedge \exists x_{n} \ldots \exists x_{1} B\left(x_{1} / \mathbf{v}_{1}, \ldots, x_{n} / \mathbf{v}_{n}, x_{n+1} / \mathbf{v}_{n+1}\right)\right)$, as $\mathbf{v}_{n+1}$ is not in $A$, so $L_{n+1} \rightarrow \exists x_{n+1} L_{n}\left(x_{n+1} / \mathbf{v}_{n+1}\right)$ is a Conjunctive $\exists$-Distribution axiom. It follows that $\vdash_{\mathrm{L}} L_{n+1} \rightarrow R_{n+1}$. Thus $\vdash_{\mathrm{L}} L_{n} \rightarrow R_{n}$ for all $n$, by induction.

THEOREM 4.2. Let $\mathrm{L}$ be a logic having the $\exists$-postulates of Figure 3. Then the canonical model $\mathfrak{A}_{\mathrm{L}}$ for $\mathrm{L}$ satisfies the condition

$$
\text { IV(i)(d): } u \geq t \downarrow \text { implies } \exists u^{+} \geq t\left(u^{+} \downarrow=u\right) .
$$

Proof. Suppose that in $\mathfrak{A}_{\mathrm{L}}$ we have $u \geq t \downarrow$ with $t=\left(\Gamma, V^{+}\right)$and $u=(\Delta, V)$. Hence $V \subseteq V^{+}$and $\Gamma \cap \operatorname{Fml}(V) \subseteq \Delta$. Let $\Gamma^{+}=\left\{C \in \operatorname{Fml}\left(V^{+}\right): \Delta \cup \Gamma \vdash_{\mathrm{L}} C\right\}$. Then $\Gamma^{+}$is an L-theory in $V^{+}$with $\Delta \cup \Gamma \subseteq \Gamma^{+}$and $\Delta \subseteq \Gamma^{+} \cap \operatorname{Fml}(V)$. We prove the converse of this last inclusion.

Let $C \in \Gamma^{+} \cap \operatorname{Fml}(V)$. Then $\Delta \cup \Gamma \vdash_{\mathrm{L}} C$, so as the theories $\Delta$ and $\Gamma$ are closed under conjunction, then either there exists $A \in \Delta$ with $\left\llcorner_{\mathrm{L}} A \rightarrow C\right.$; or there exists $B \in \Gamma$ with $\vdash_{\mathrm{L}} B \rightarrow C$; or there exists $A \in \Delta$ and $B \in \Gamma$ with $\vdash_{\llcorner} A \wedge B \rightarrow C$. We take the last case first.

Let $V^{+}-V=\left\{\mathbf{v}_{1}, \ldots, \mathbf{v}_{n}\right\}$, and take new $x_{1}, \ldots, x_{n}$ not occurring in $A, B, C$. Since the $\mathbf{v}_{i}$ do not occur in $C$ or in $A$, from $\left\llcorner_{\mathrm{L}} A \wedge B \rightarrow C\right.$ by $n$ applications of $\exists$-Elimination we get

$$
\vdash \exists x_{n} \ldots \exists x_{1}\left(A \wedge B\left(x_{1} / \mathbf{v}_{1}, \ldots, x_{n} / \mathbf{v}_{n}\right)\right) \rightarrow C .
$$

Let $B^{*}=\exists x_{n} \ldots \exists x_{1} B\left(x_{1} / \mathbf{v}_{1}, \ldots, x_{n} / \mathbf{v}_{n}\right) \in \operatorname{Fml}(V)$. Then $\vdash_{\mathrm{L}} A \wedge B^{*} \rightarrow C$ by Lemma 4.1(2) and the last result. Now $B \in \Gamma$, and $\left\llcorner B \rightarrow B^{*}\right.$ by Lemma 4.1(1), so $B^{*} \in$ $\Gamma \cap \operatorname{Fml}(V) \subseteq \Delta$. We now have $A, B^{*} \in \Delta$, and $\vdash_{\mathrm{L}} A \wedge B^{*} \rightarrow C$, giving $C \in \Delta$ as required because $C \in \operatorname{Fml}(V)$.

In the case $\vdash_{\mathrm{L}} A \rightarrow C$ with $A \in \Delta$, then we get $C \in \Delta$ immediately. If however we have $\vdash_{\llcorner} B \rightarrow C$ with $B \in \Gamma$, then $\vdash_{\llcorner} B^{*} \rightarrow C$ follows directly by $\exists$-Elimination, and again $C \in \Delta$.

This establishes that $\Gamma^{+} \cap \operatorname{Fml}(V)=\Delta$. Putting $u^{+}=\left(\Gamma^{+}, V^{+}\right)$, we then get $u^{+} \geq t$ and $u^{+} \downarrow_{V}=u$.

Note that in this proof, since $\Gamma \subseteq \Gamma^{+}$, we get $\operatorname{Thm}\left(u^{+}\right) \neq \emptyset$ if $\operatorname{Thm}(t) \neq \emptyset$, as required for the canonical models of the identity systems of Section 3 . Thus all the logics BQX $\tau \approx$, 
as well as the BQX's, are characterized by models satisfying the condition IV(i)(d). We will assume this condition in the next section.

§5. Conservatively adding $\supset$. The language with $\tau$ and $\approx$ will now be expanded further to include a new binary connective $\supset$. Let $\mathfrak{A}$ be a stratified identity model with truth relation $\models$ for the language without $\supset$. Assume that $\mathfrak{A}$ satisfies IV(i)(d). Define a new truth relation $\models^{\prime}$ on $\mathfrak{A}$ for the language including $\supset$, by inductively putting

$$
t \models^{\prime} B \supset C \text { iff for all } u \geq t, u \models^{\prime} B \text { implies } u \models^{\prime} C,
$$

and otherwise defining $\models^{\prime}$ by exactly the same conditions as for $\models$ given earlier.

Lemma 5.1. For any sentence A, the Truth Lemmas 2.2 hold for A under $\models^{\prime}$ in $\mathfrak{A}$.

Proof. By induction on the length of $A$. The only new case is $A=B \supset C$.

Truth Extension: Let $t \models^{\prime} B \supset C$ and $t \leq v$. If $u \geq v$ and $u \models^{\prime} B$, then $u \geq t$ so by (5.1) $u \models \models^{\prime} C$. This shows that $v \models^{\prime} B \supset C$, by (5.1) again.

Truth Across: Let $A^{\prime}$ be an $i j$-variant of $A=B \supset C$. Then $A^{\prime}=B^{\prime} \supset C^{\prime}$, where $B^{\prime}$ and $C^{\prime}$ are $i j$-variants of $B$ and $C$. Let $t={ }^{i j} \vec{t}$ and $t \models^{\prime} B \supset C$. If $t \leq u$ and $u \models^{\prime} B^{\prime}$, then by Lemma 3.1(3) $u={ }^{i j} \vec{u}$, and so $u \models^{\prime} B$ by induction hypothesis, as $B$ is an $i j$ variant of $B^{\prime}$. Hence by (5.1) $u \models^{\prime} C$, so $u \models \models^{\prime} C^{\prime}$ by induction hypothesis. This shows that $t \models^{\prime} B^{\prime} \supset C^{\prime}$ as required.

Truth Down: Let $t \downarrow \models^{\prime} B \supset C$. If $t \leq u$ and $u \models^{\prime} B$, then $t \downarrow \leq u \downarrow$ (III(i)(b)), and $u \downarrow \models^{\prime} B$ by induction hypothesis on $B$, hence $u \downarrow \models^{\prime} C$ by (5.1), and so $u \models^{\prime} C$ by hypothesis on $C$. This shows that $t \models^{\prime} B \supset C$.

Conversely, let $t \models^{\prime} B \supset C$. If $t \downarrow \leq u$ and $u \models^{\prime} B$, then by IV(i)(d) there exists $u^{+} \geq t$ with $u^{+} \downarrow=u$, hence $u^{+} \models^{\prime} B$ by hypothesis on $B$. Then $u^{+} \models^{\prime} C$ by (5.1), and so $u \models^{\prime} C$ by hypothesis on $C$. This shows that $t \downarrow \models^{\prime} B \supset C$.

Truth Saturation: If $t \forall^{\prime} B \supset C$, then for some $u \geq t, u \models^{\prime} B$ and $u \nvdash^{\prime} C$. By induction hypothesis, there exists $a \geq u$ such that $a \forall^{\prime} C$. Then $a \models^{\prime} B$ by Truth Extension. But then $a \geq t$ and $a \not \forall^{\prime} B \supset C$.

Now let $\mathrm{HBQX} \tau \approx$ be the logic defined by all the postulates of $\mathrm{BQX} \tau \approx$ stated for all formulas in the new expanded language with $\supset$, and the $\wedge$-Residuation rules

$$
\frac{A \wedge B \rightarrow C}{A \rightarrow(B \supset C)} \quad \frac{A \rightarrow(B \supset C)}{A \wedge B \rightarrow C}
$$

THEOREM 5.2. If $X$ is any set of the standard postulates of Figure 2, then the logic $H B Q X \boldsymbol{\tau} \approx$ is a conservative extension of $B Q X \boldsymbol{\tau} \approx$.

Proof. By the method used to prove Theorem 2.5.

Let $\mathrm{L}=\mathrm{BQX}{ }^{\tau} \approx$, and suppose $B$ is a $\supset$-free formula such that $\nvdash_{\mathrm{L}} B$. By Theorem 3.5, there is a (canonical) stratified identity model $\mathfrak{A}$ satisfying the structure conditions for $\mathrm{X}$, and having $\mathfrak{A} \forall \forall$. We can assume $\mathfrak{A}$ satisfies $\operatorname{IV}(\mathrm{i})(\mathrm{d})$ (Theorem 4.2). Extend $\models$ as above to a relation $\models^{\prime}$ on $\mathfrak{A}$ for the language including $\supset$.

Given that $\mathfrak{A}$ satisfies the structure conditions for $\mathrm{X}$, and the Truth Lemmas all hold in $\left(\mathfrak{A}, \models^{\prime}\right)$ for the expanded language (Lemma 5.1), earlier soundness arguments apply to show that all postulates of $\mathrm{BQX}{ }^{\boldsymbol{\tau} \approx}$ are sound in $\left(\mathfrak{A}, \models^{\prime}\right)$. In addition, the residuation rules are sound: it is readily shown, with the help of Corollary 2.3(4) especially, that

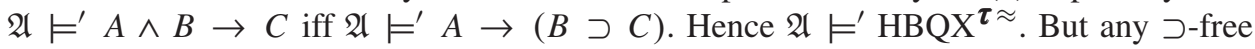
formula $A$ has $t \models A$ iff $t \models^{\prime} A$, and hence $\mathfrak{A} \nvdash^{\prime} B$. Therefore HBQX $\boldsymbol{\tau} \approx \nvdash B$ as required. 
§6. Antitone and contrapositive negation. Points of a model may be thought of as states containing certain information, with $t \models A$ meaning that $t$ contains the information that $A$. There is an informational interpretation of negation that takes $t \models \neg A$ to mean that only states "incompatible" with $t$ contain the information that $A$, or equivalently that no state compatible with $t$ contains this information (Dunn, 1993). Then $t \models \neg \neg A$ asserts that any state compatible with $t$ is compatible with a state containing the information that $A$. It is not clear that it should follow that $t$ itself contains the information that $A$, so this interpretation apparently does not validate Double-Negation Elimination.

In this section our results will be extended to logics that lack Double-Negation Elimination. Here it is no longer appropriate to use negation to define $\vee$ or $\exists$, so all of $\tau, \wedge, \vee$, $\neg, \rightarrow, \forall$, and $\exists$ will be taken as primitive, and we need new semantics for $\vee, \neg$, and $\exists$. In addition, a primitive binary connective $\circ$ (fusion) will be added, to be interpreted via the operation $t \cdot u$ in structures. The co-theory function is dropped in favor of a binary incompatibility relation $\perp$ which is used to model $\neg$ in a manner first introduced in Goldblatt (1974).

A structure now has the form

$$
\mathfrak{A}=(T, S, D, l, \cdot, \perp, \geq, \uparrow, \downarrow, \rightarrow, \varphi),
$$

with the function $-: S \rightarrow S$ replaced by a binary relation $\perp$ on $T$ such that $t \perp u$ implies $t \equiv u$.

The structural conditions on a model are adapted as follows:

I. Standard: (v) and (vi), which refer to - , are replaced by

(v) $u \geq t$ and $t \perp v$ implies $u \perp v$.

(vi)' $(\forall a \geq t)(a \perp u)$ implies $t \perp u$.

Also we add

(ix) $\exists u(a \not \perp u) \quad$ [equivalently: $\forall u(t \perp u)$ implies $t \notin S$ ].

II. Levels: (iii) is strengthened to

(iii) $\forall \alpha, \beta, \gamma(\alpha \cup(\gamma-\beta) \in \mathfrak{D})$.

IV. Interaction of $\uparrow$ and $\downarrow$ : (ii) is replaced by

(ii)' (With $\perp$ )

(a) $t \downarrow \perp u \downarrow$ implies $t \perp u$.

(b) $t \perp u \uparrow$ implies $t \downarrow \perp u$.

IV(i)(b) is withdrawn: it can be optionally added for logics that have Disjunctive $\forall$-Distribution. IV(i)(d) is also withdrawn: it can be optionally added for logics that have Conjunctive $\exists$-Distribution.

V. Behavior of $\rightarrow$ : (v) is replaced by

(v) $t \perp \vec{u}$ implies $\vec{t} \perp u$,

and (vi) is strengthened to

(vi) $\overrightarrow{(t u)}=\vec{t} \vec{u} \leq \vec{t} u$. 
Also we add

(x) $\overrightarrow{(t \downarrow)} \geq(\vec{t}) \downarrow$, where $\rightarrow$ is ${ }^{i j} \rightarrow$ with $i, j \in D_{t \downarrow}$.

All other model conditions remain unchanged. We will call a structure satisfying these modified conditions for groups I-V an MQ-model. Here MQ refers to a logic to be defined below, and the "M" is for "minimal".

Note that the new $\mathrm{V}(\mathrm{x})$ can be strengthened to an equality: using III(iii)(a), $\mathrm{V}$ (vii), III(iii)(b), and the monotonicity of $(\overrightarrow{)})$ and $\downarrow$, we get $\overrightarrow{(t \downarrow)}=\overrightarrow{(t \downarrow)} \uparrow \downarrow=\overrightarrow{(t \downarrow \uparrow)} \leq \leq(\vec{t}) \downarrow$.

The truth relation $\models$ on a model of this kind is defined by the previous clauses for atomic sentences, $\wedge, \rightarrow$, and $\forall$, and the new

- $t \models \neg B$ iff $(\forall u \equiv t)(u \models B$ implies $t \perp u)$.

- $t \models B \vee C$ iff $(\forall a \geq t)(a \models B$ or $a \models C)$.

- $t \models B \circ C$ iff $(\forall a \geq t)(\exists u, v)(a \geq u v$ and $u \models B$ and $v \models C)$.

- $t \models \exists x B$ iff $(\forall a \geq t)(\forall \rho \in \mathfrak{D})(\exists u)\left(\exists i \in D_{u}-\rho\right)\left(u \models B(i / x)\right.$ and $\left.u \downarrow_{a} \leq a\right)$.

In the clause for $\exists$ it is implicit that $D_{a} \subseteq D_{u}$. This complex clause seems to be required to derive the Truth Lemmas, especially the "up" direction of Truth Down for $\exists$. But once these are established, we will show, in Corollary 6.2, that the clause is equivalent to the simpler and more intuitive (2.1).

LEMMA 6.1. The Truth Lemmas 2.2 hold for any sentence $A$ in the present language under the truth relation in an MQ-model.

Proof. It is a general fact that if a condition $\theta(t)$ is defined by, or equivalent to, one of the form $(\forall a \geq t) \psi(a / t)$, then it satisfies Extension: $\theta(t)$ and $t \leq u$ implies $\theta(u / t)$; and Saturation: $(\forall a \geq t) \theta(a / t)$ implies $\theta(t)$. Thus Truth Extension and Truth Saturation hold when $A$ has any of the forms $B \vee C, B \circ C$, and $\exists x B$, just by the way truth is defined for these cases. We consider the remaining new cases in turn.

Truth Extension: Let $t \models \neg B$ and $t \leq u$. Then if $u \models B, t \perp v$ by the semantics of $\neg$, and therefore $u \perp v$ by $\mathrm{I}(\mathrm{v})^{\prime}$. This shows that $u \models \neg B$.

Truth Across: Let $t={ }^{i j} \vec{t}$. As usual, $A^{\prime}$ denotes an $i j$-variant of $A$. If $A$ is of the form $\neg B, B \vee C, B \circ C$, or $\exists x B$, then $A^{\prime}$ is of the form $\neg B^{\prime}, B^{\prime} \vee C^{\prime}, B^{\prime} \circ C^{\prime}$, or $\exists x B^{\prime}$, where $B^{\prime}$ and $C^{\prime}$ are $i j$-variants of $B$ and $C$.

For the negation case, suppose $t \models \neg B$. If $u \models B^{\prime}$, then $\vec{u} \models B^{\prime}$ by Truth Extension as $u \leq \vec{u}$; so $\vec{u} \models B$ by induction hypothesis as $B$ is an $i j$-variant of $B^{\prime}$; hence $t \perp \vec{u}$ as $t \models \neg B$; so $\vec{t} \perp u$ by $\mathrm{V}(\mathrm{v})^{\prime}$. This shows $\vec{t} \models \neg B^{\prime}$. But $\vec{t}=t$.

For disjunction, suppose $t \models B \vee C$. Let $a \geq t$. Then by $\mathrm{V}$ (iv) there exists $a^{\prime}$ with $t \leq a^{\prime} \leq a$ and $a^{\prime}=a^{\prime}$. Then as $t \models B \vee C$, either $a^{\prime} \models B$ or $a^{\prime} \vDash C$; hence $a^{\prime} \models B^{\prime}$ or $a^{\prime} \models C^{\prime}$ by induction hypothesis as $a^{\prime}=\overrightarrow{a^{\prime}}$; so $a \models B^{\prime}$ or $a \models C^{\prime}$ by Truth Extension. This shows $t \models B^{\prime} \vee C^{\prime}$.

For fusion, suppose $t \models B \circ C$. Let $a \geq t$. Take $a^{\prime}$ with $t \leq a^{\prime} \leq a$ and $a^{\prime}=\vec{a}^{\prime}$. Then there exist $u, v$ with $a^{\prime} \geq u v, u \models B$, and $v \models C$. As $u \leq \vec{u}$, by Truth Extension and induction hypothesis we then get $\vec{u} \models B^{\prime}$. Similarly $\vec{v} \models C^{\prime}$. But using the new part of $\mathrm{V}(\mathrm{vi})^{\prime}, \vec{u} \vec{v}=\overrightarrow{(u v)} \leq \vec{a}^{\prime}=a^{\prime} \leq a$. This is enough to ensure $t \models B^{\prime} \circ C^{\prime}$.

For the case of $\exists$, let $t \models \exists x B$. Take any $a \geq t$ and $\rho \in \mathfrak{D}$. By V(iv) again take $a^{\prime}$ with $t \leq a^{\prime} \leq a$ and $a^{\prime}=\vec{a}^{\prime}$. From $t \models \exists x B$, there exists a $u$ and a $k \in D_{u}-\rho$ with $u \models B(k / x)$ and $u \downarrow_{a^{\prime}} \leq a^{\prime}$. By Truth Extension and induction hypothesis, $\vec{u} \models B^{\prime}(k / x)$. 
But using the new $\mathrm{V}(\mathrm{x})$,

$$
(\vec{u}) \downarrow_{a}=(\vec{u}) \downarrow_{a^{\prime}} \leq \overrightarrow{\left(u \downarrow_{a^{\prime}}\right)} \leq \overrightarrow{a^{\prime}}=a^{\prime} \leq a .
$$

Since $k \in D_{\vec{u}}-\rho$ and $\vec{u} \models B^{\prime}(k / x)$, this established the necessary condition for $t \models \exists x B^{\prime}$.

Truth Down: For the negation case, let $t \downarrow \models \neg B$. Then if $t \equiv u \models B$, by induction hypothesis $u \downarrow \models B$, hence $t \downarrow \perp u \downarrow$ by the semantics of $\neg$, and so $t \perp u$ by the new IV(ii)'(a). This shows $t \models \neg B$. Conversely, let $t \models \neg B$. Then if $t \downarrow \equiv u \models B$, by induction hypothesis $u \uparrow \models B$ as $u \uparrow \downarrow=u$, hence $t \perp u \uparrow$ as $t \models \neg B$, so $t \downarrow \perp u$ by IV(ii)' (b). This shows $t \downarrow \models \neg B$.

For disjunction, suppose $t \downarrow \models B \vee C$. Let $a \geq t$. Then $a \downarrow \geq t \downarrow$ and $a \downarrow \in S$, so $a \downarrow \models B$ or $a \downarrow \models C$, hence $a \models B$ or $a \models C$ by induction hypothesis. This shows $t \models B \vee C$. Conversely, suppose $t \models B \vee C$ and $a \geq t \downarrow$. By IV(i)(c) there exists $a^{+} \geq t$ with $a^{+} \downarrow \leq a$. Then $a^{+} \models B$ or $a^{+} \models C$, so $a^{+} \downarrow \models B$ or $a^{+} \downarrow \models C$ by induction hypothesis, hence $a \downarrow \models B$ or $a \downarrow \models C$ by Truth Extension. This shows $t \downarrow \models$ $B \vee C$.

For fusion, suppose $t \downarrow \models B \circ C$. Let $a \geq t$. Then $a \downarrow \geq t \downarrow$, so there exist $u$, $v$ with $a \downarrow \geq u v, u \models B$, and $v \models C$. Then $u \uparrow \models B$, and $v \uparrow \models C$ by induction hypothesis, as $u \uparrow \downarrow=u$ and $v \uparrow \downarrow=v$. But using IV(iii)(a), $u \uparrow v \uparrow=(u v) \uparrow \leq(a \downarrow) \uparrow \leq a$ (III(iii)(b)). This shows $t \models B \circ C$. Conversely, if $t \models B \circ C$ and $a \geq t \downarrow$, take an $a^{+} \geq t$ with $a^{+} \downarrow \leq a$, by IV(i)(c). Then there exist $u, v$ with $a^{+} \geq u v, u \models B$, and $v \models C$. Hence $u \downarrow \models B$, and $v \downarrow \models C$ by induction hypothesis. Now (uv) $\geq \geq u \downarrow v \downarrow$ [SQL, Lemma 1(i)], so we have $a \geq a^{+} \downarrow \geq(u v) \downarrow \geq u \downarrow v \downarrow$, hence $a \geq u \downarrow v \downarrow$. This shows $t \downarrow \models B \circ C$.

For the $\exists$ case, suppose first that $t \models \exists x B$. To show $t \downarrow \models \exists x B$, take any $a \geq t \downarrow$ and $\rho \in \mathfrak{D}$. By IV(i)(c) there exists $a^{+} \geq t$ with $a^{+} \downarrow_{a} \leq a$. Hence there exist a $u$ and an $i \in D_{u}-\rho$ such that $u \models B(i / x)$ and $u \downarrow_{a^{+}} \leq a^{+}$. But then using III(ii)(b), $u \downarrow_{a}=u \downarrow_{a^{+}} \downarrow_{a} \leq a^{+} \downarrow_{a} \leq a$, giving $u \downarrow_{a} \leq a$ as required.

Conversely, let $t \downarrow \models \exists x B$. To show $t \models \exists x B$, take any $a \geq t$ and $\rho \in \mathfrak{D}$. This is the most demanding case: we have to find a $u$ and an $i \notin \rho$ with $u \models B(i / x)$ and $u \downarrow \leq a$. First, let $\alpha=D_{t \downarrow}$ and $\beta=D_{t}=D_{a}$. Using II(ii), take a $\rho^{*} \in \mathfrak{D}$ with $\beta \cup \rho \subseteq \rho^{*}$. Then $t \downarrow \leq a \downarrow \in S$ and $t \downarrow \models \exists x B$, so there exists a $v$ with $\alpha \subseteq D_{v}$ and an $i \in D_{v}-\rho^{*}$ such that $v \models B(i / x)$ and $v \downarrow_{\alpha} \leq a \downarrow_{\alpha}$.

Now let $\gamma=D_{v}$, and take a $\delta \in \mathfrak{D}$ with $\gamma \cup \beta \subseteq \delta$. We have $\alpha \subseteq \beta \cap \gamma$, and $i \in \gamma-\beta$ as $\beta \subseteq \rho^{*}$ and $i \notin \rho^{*}$. Then as $\rho \subseteq \rho^{*}, i \notin \rho$. Put $\sigma=\alpha \cup(\gamma-\beta)$, which belongs to $\mathfrak{D}$ by the new II(iii) ${ }^{\prime}$. [It may help to draw a Venn diagram showing the relationships between $\alpha, \beta, \gamma, \delta, \sigma$, and $i$.]

Next let $s=v \downarrow_{\sigma}$. Then $s \models B(i / x)$ by induction hypothesis, as $v \models B(i / x)$. Hence $s \uparrow^{\delta} \models B(i / x)$ by induction hypothesis, as $s \uparrow^{\delta} \downarrow_{\sigma}=s$. So putting $u=s \uparrow^{\delta}$ it remains only to show that $u \downarrow_{\beta} \leq a$. Now

$$
D_{s} \cap D_{s \downarrow_{\alpha} \uparrow^{\beta}}=\sigma \cap \beta=[\alpha \cup(\gamma-\beta)] \cap \beta=\alpha=D_{s \downarrow_{\alpha}},
$$

so by III(iii)(c), $s \downarrow_{\alpha} \uparrow^{\beta}=s \uparrow^{\delta} \downarrow_{\beta}$. Hence

$$
u \downarrow_{\beta}=s \uparrow^{\delta} \downarrow_{\beta}=s \downarrow_{\alpha} \uparrow^{\beta}=v \downarrow_{\sigma} \downarrow_{\alpha} \uparrow^{\beta}=v \downarrow_{\alpha} \uparrow^{\beta} \leq a \downarrow_{\alpha} \uparrow^{\beta} \leq a
$$

as required.

Truth Saturation: The only case left unresolved is $A=\neg B$. Suppose that $(\forall a \geq$ $t)(a \models \neg B)$. If $u \equiv t$ and $u \models B$, then $(\forall a \geq t)(a \perp u)$ by the semantics of $\neg$, so $t \perp u$ by the new I(vi) $)^{\prime}$. This proves $t \models \neg B$, as required. 
COROLLARY 6.2. In any MQ-model, the following are equivalent.

(1) $t \models \exists x B$.

(2) $(\forall a \geq t)(\exists u)\left(\exists i \in D_{u}\right)\left(u \models B(i / x)\right.$ and $\left.u \downarrow_{a} \leq a\right)$.

(3) $(\forall a \geq t)(\exists b)\left(\exists i \in D_{b}\right)\left(b \models B(i / x)\right.$ and $\left.b \downarrow_{a} \leq a\right)$. [see (2.1)]

Proof. (1) implies (2): Given $a \geq t$, just put $\rho=D_{a}$ in the condition for (1) to conclude there exists a $u$ and an $i \in D_{u}-D_{a}$ with $u \models B(i / x)$ and $u \downarrow_{a} \leq a$. Hence (2) holds.

(2) implies (3): Let $a \geq t$. From (2) we obtain $u$ and $i$ with $u \models B(i / x)$ and $u \downarrow_{a} \leq a$. By IV(i)(c) there exists $a^{+} \geq u$ with $a^{+} \downarrow_{a} \leq a$. Then $a^{+} \models B(i / x)$ by Truth Extension, so $b=a^{+}$fulfills (3).

(3) implies (1): Let $a \geq t$ and $\rho \in \mathfrak{D}$. From (3) we obtain $b$ and $i$ with $b \models B(i / x)$ and $b \downarrow_{a} \leq a$. Now by II(i,ii), take a $\beta \in \mathfrak{D}$ with $\rho \cup D_{b} \subset \beta$, and pick $j \in \beta-\left(\rho \cup D_{b}\right)$. Thus $j \in \beta-\rho$. Then $b \uparrow^{\beta} \models B(i / x)$ by Truth Down, as $b \uparrow^{\beta} \downarrow_{b}=b$. Let $\rightarrow$ be $^{i j \rightarrow}$ and put $u=\overrightarrow{b \uparrow}$, where $\uparrow$ is $\uparrow^{\beta}$. Then $u \models B(i / x)$ by Truth Extension, hence $u \models B(j / x)$ by Truth Across. Now $u \downarrow_{b}=\overrightarrow{b \uparrow} \downarrow_{b} \leq b$ by V(viii) as $j \in D_{b \uparrow}-D_{b}$ and $i \in D_{b}$, so $u \downarrow_{a}=u \downarrow_{b} \downarrow_{a} \leq b \downarrow_{a} \leq a$. Thus we have found a $u$ and $j \in D_{u}-\rho$ with $u \models B(j / x)$ and $u \downarrow_{a} \leq a$, as required to show $t \models \exists x B$, that is (1).

Using Truth Extension, results (2)-(4) of Corollary 2.3 can be proved for the present semantics (see also [MFE, p. 350]).

Figure 4 lists axioms and rules for a system MQ. This has the postulates for $\tau$, and all the quantifier-free postulates of Figure 1 except the Double-Negation Elimination axiom and the Contraposition rule. The new Antitonicity rule is the only postulate for negation. There are new Disjunction Introduction axioms, as $\vee$ is now primitive and these are independent. The Fusion-Residuation rules are also new. Note that Disjunctive $\forall$-Distribution (Figure 1) and Conjunctive $\exists$-Distribution (Figure 3) are not included in MQ. The effect of adding these two distribution principles to MQ will be considered below (see especially Remark 6.8 and its preceding two paragraphs, as well as part (2) of the summary in Section 8).

A logic for the present language is a set $\mathrm{L}$ of real formulas closed under the postulates of Figure 4. A quasi-logic has all of these postulates except the rules of Generalization and $\exists$-Elimination. MQ is the smallest logic.

LEMMA 6.3.

(1) $\vdash_{\mathrm{L}} \neg A_{1} \vee \cdots \vee \neg A_{n} \rightarrow \neg\left(A_{1} \wedge \cdots \wedge A_{n}\right)$, for any quasi-logic $\mathrm{L}$

(2) The Fusion-Residuation rules together are equivalent, over the postulates for relevant implication, to the combination of the schemas

T1: $(A \rightarrow B) \circ A \rightarrow B$

$\mathrm{T} 2: A \rightarrow(B \rightarrow A \circ B)$

with the Fusion-Monotonicity rule

$$
\frac{A \rightarrow A^{*}, B \rightarrow B^{*}}{A \circ B \rightarrow A^{*} \circ B^{*}} .
$$

Proof. (1): By the Conjunction Elimination axioms and the Antitonicity rule, $\vdash_{\llcorner} \neg A_{i} \rightarrow$ $\neg\left(A_{1} \wedge A_{2}\right)$ for $i=1,2$, so by Disjunction Elimination $\vdash_{\mathrm{L}} \neg A_{1} \vee \neg A_{2} \rightarrow \neg\left(A_{1} \wedge A_{2}\right)$. The general case of $n$ is left to the reader. 
Primitives: $\tau, \wedge, \vee, \neg, \rightarrow, \circ, \forall, \exists$

\section{Axioms}

$$
\begin{aligned}
& \tau \\
& A \rightarrow A \\
& A \wedge B \rightarrow A, A \wedge B \rightarrow B \\
& (A \rightarrow B) \wedge(A \rightarrow C) \rightarrow(A \rightarrow B \wedge C) \\
& A \rightarrow A \vee B, \quad B \rightarrow A \vee B \\
& (A \rightarrow C) \wedge(B \rightarrow C) \rightarrow(A \vee B \rightarrow C) \\
& A \wedge(B \vee C) \rightarrow(A \wedge B) \vee(A \wedge C) \\
& \forall x A \rightarrow A(\mathbf{v} / x) \\
& \forall x(A \rightarrow B) \rightarrow(A \rightarrow \forall x B), \text { with } x \text { not free in } A . \\
& A(\mathbf{v} / x) \rightarrow \exists x A
\end{aligned}
$$

$\tau$-Axiom
Identity
Conjunction Elimination
Conjunction Introduction
Disjunction Introduction
Disjunction Elimination
$\wedge \vee$-Distribution
Specification
Relevant $\forall$-Distribution
Existence

Rules

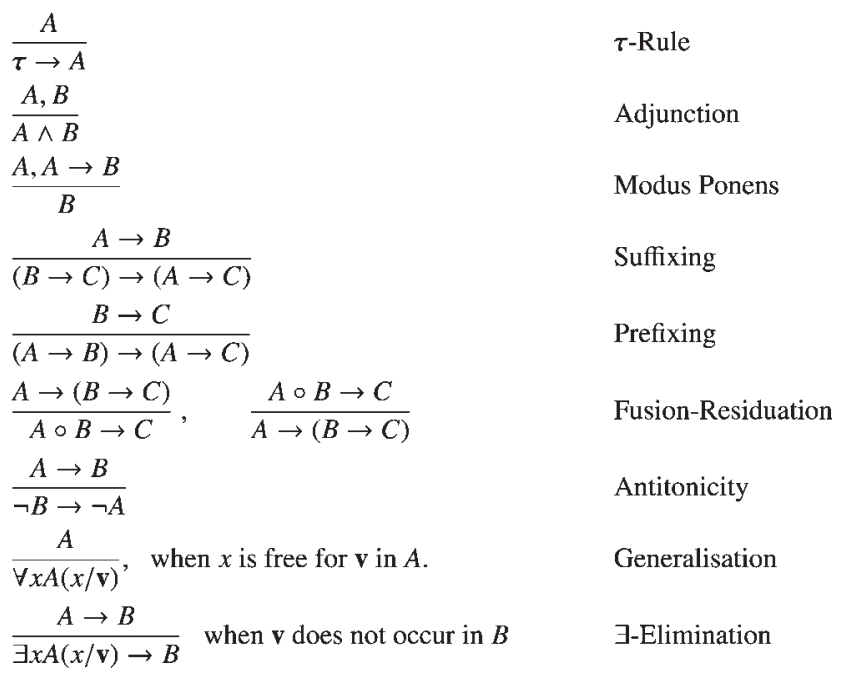

Fig. 4. Axioms and rules for MQ.

(2): T1 follows from $(A \rightarrow B) \rightarrow(A \rightarrow B)$ by the first Fusion-Residuation rule, and T2 follows from $A \circ B \rightarrow A \circ B$ by the second rule. The derivation of Fusion-Monotonicity is lengthier but routine, and is left to the reader, as is the converse direction of (2).

\section{THEOREM 6.4 (SOUNDNESS).}

(1) The MQ-theorems are valid in all MQ-models.

(2) Conjunctive $\exists$-Distribution is valid in any model satisfying

$$
\mathrm{IV}(\mathrm{i})(\mathrm{d}): \quad u \geq t \downarrow \text { implies } \exists u^{+} \geq t\left(u^{+} \downarrow=u\right) .
$$

Proof. Using (2)-(4) of Corollary 2.3, it is straightforward to verify that the Disjunction axioms are valid under the present semantics. We go through the verification for the other additional axioms.

Antintonicity axiom: Let $A \rightarrow B$ be true in a model $\mathfrak{A}$. If $t \models \neg B$, then for $u \equiv t$, if $u \models A$, then $u \models B$ as $\mathfrak{A} \models A \rightarrow B$, hence $t \perp u$ by the semantics of $\neg$. Hence $t \models \neg A$. This shows $\mathfrak{A} \models \neg B \rightarrow \neg A$.

Fusion-Residuation rules: For the first rule, let $\mathfrak{A} \models A \rightarrow(B \rightarrow C)$. Suppose $t \models$ $A \circ B$. We want $t \models C$ to conclude that $\mathfrak{A} \models A \circ B \rightarrow C$. Now for any $a \geq t$, there 
exist $u, v$ with $u v \leq a, u \models A$ and $v \models B$. Then $u \models B \rightarrow C$ as $\mathfrak{A} \models A \rightarrow(B \rightarrow C)$, so $u v \models C$ as $u \models A$. As $u v \leq a$, this gives $a \models C$ by Truth Extension. This shows $(\forall a \geq t)(a \models C)$, therefore $a \models C$ as required, by Truth Saturation.

For the second rule, let $\mathfrak{A} \models A \circ B \rightarrow C$. Suppose $t \models A$. We want $t \models(B \rightarrow C)$ to conclude that $\mathfrak{A} \models A \rightarrow(B \rightarrow C)$. So let $u \equiv t$ have $u \models B$. We want $t u \models C$. But now for all $a \geq t u$ we have $t \models A$ and $u \models B$, which shows that $t u \models A \circ B$ by the semantics of $\circ$. Hence $t u \models C$ as $\mathfrak{A} \models A \circ B \rightarrow C$.

Existence axiom: Let real formula $B$ have the form $A(\mathbf{v} / x) \rightarrow \exists x A$. We have to show that every sentence obtained by specifying the values of the real variables of $B$ to be particular constants from $I$ is true in $\mathfrak{A}$. We may suppose that all such variables except $\mathbf{v}$ are already specified. So we take any designated point $l$ of $\mathfrak{A}$, and show that $l \models B(i / \mathbf{v})$ if $i \in D_{l}$. Equivalently, for any $t \equiv l, t \models A(\mathbf{v} / x)(i / \mathbf{v})$ implies $t \models \exists x A(i / \mathbf{v})$. But notice that $A(\mathbf{v} / x)(i / \mathbf{v})$ is the same formula $A(i / \mathbf{v})(i / x)$ : in both cases we get the formula resulting from replacing all occurrences of $x$ and $\mathbf{v}$ in $A$ by $i$.

Now if $t \models A(i / \mathbf{v})(i / x)$, then for any $a \geq t$, putting $u=a$ we get $u \models A(i / \mathbf{v})(i / x)$ by Truth Extension, and $u \downarrow_{a} \leq a$, which verifies that $t \models \exists x A(i / \mathbf{v})$ by Corollary 6.2(2).

$\exists$-Elimination rule: Let real formula $A \rightarrow B$ be true in $\mathfrak{A}$, with $\mathbf{v}$ its only real variable, and $\mathbf{v}$ not in $B$. Let the sentence $\exists x A(x / \mathbf{v}) \rightarrow B$ be defined at $t$, and suppose $t \models$ $\exists x A(x / \mathbf{v})$. Then by Corollary 6.2(2), given any $a \geq t$ there exist $u$ and $i \in D_{u}$ with $u \models A(x / \mathbf{v})(i / x)$ and $u \downarrow_{a} \leq a$. Since $A$ has no free $x, A(x / \mathbf{v})(i / x)$ is just $A(i / \mathbf{v})$, so $u \models A(i / \mathbf{v})$. But $\mathfrak{A} \models A(i / \mathbf{v}) \rightarrow B$, so $u \models B$. Now $B \in \operatorname{Sen}(t)=\operatorname{Sen}(a)$, so $u \downarrow_{a} \models B$ by Truth Down, hence $a \models B$ by Truth Extension. Altogether this shows that $(\forall a \geq t)(a \models B)$, hence $t \models B$ by Truth Saturation. In sum, we showed that in general $t \models \exists x A(x / \mathbf{v})$ implies $t \models B$, hence $\exists x A(x / \mathbf{v}) \rightarrow B$ is true in $\mathfrak{A}$.

That concludes the proof of (1). For (2), suppose $A \wedge \exists x B \in \operatorname{Sen}(t)$ and $t=A \wedge \exists x B$. Then for any $a \geq t$, since $t \models \exists x B$, there exist $u$ and $i \in D_{u}$ such that $u \models B(i / x)$ and $u \downarrow \leq a$. As $t \models A$, then $a \models A$ by Truth Extension. As $a \geq u \downarrow$, if $I V(i)(d)$ holds then there is some $v \geq u$ with $v \downarrow=a$. Hence $v \models A$ by Truth Down. Also $v \models B(i / x)$ by Truth Extension. Now we have $v \models A \wedge B(i / x)$. But $A \wedge B(i / x)=(A \wedge B)(i / x)$ as $x$ is not free in $A$. So we have shown that $(\forall a \geq t)(\exists u, i)(u \models(A \wedge B)(i / x)$ and $u \downarrow \leq a)$. This ensures $t \models \exists x(A \wedge B)$. Thus IV(i)(d) guarantees that $A \wedge \exists x B \rightarrow \exists x(A \wedge B)$ is true in $\mathfrak{A}$ when $x$ is not free in $A$.

To prove completeness, we associate with each logic $\mathrm{L}$ a canonical model $\mathfrak{A}_{\mathrm{L}}$ by the constructions of Section 3. The one change we need to make is to replace the co-theory function by a binary relation $t \perp u$. This is to hold only when $\operatorname{Var}(t)=\operatorname{Var}(u)$, and for such $t, u$ is defined by

$$
t \perp u \quad \text { iff } \quad \exists A \in \operatorname{Thm}(u)(\neg A \in \operatorname{Thm}(t)) .
$$

LEMMA 6.5. $\mathfrak{A}_{\mathrm{L}}$ is an MQ-model.

Proof. We verify that $\mathfrak{A}_{\mathrm{L}}$ satisfies all the new MQ-model conditions.

$\mathbf{I}(\mathbf{v})^{\prime}:$ if $u \geq t$ and $t \perp v$, there is some $A \in \operatorname{Thm}(v)$ with $\neg A \in \operatorname{Thm}(t)$. But $\operatorname{Thm}(t) \subseteq$ $\operatorname{Thm}(u)$, so $\neg A \in \operatorname{Thm}(u)$, showing $u \perp v$.

I(vi)' : Suppose $(\forall a \geq t)(a \perp u)$. We want $t \perp u$. Let $\Gamma$ be the closure under disjunction of the set $\{\neg A: A \in \operatorname{Thm}(u)\}$. It suffices to show that $\operatorname{Thm}(t) \cap \Gamma \neq \varnothing$. For if $\neg A_{1} \vee$ $\cdots \vee \neg A_{n} \in \operatorname{Thm}(t)$ with $A_{1}, \ldots, A_{n} \in \operatorname{Thm}(u)$, then using Lemma 6.3(1) we get $\neg\left(A_{1} \wedge\right.$ $\left.\cdots \wedge A_{n}\right) \in \operatorname{Thm}(t)$, so as $A_{1} \wedge \cdots \wedge A_{n} \in \operatorname{Thm}(u)$ we get $t \perp u$ as desired. But if we $\operatorname{had} \operatorname{Thm}(t) \cap \Gamma=\emptyset$, then as $\Gamma$ is closed under disjunction we could use Lindenbaum's 
Lemma to extend $\operatorname{Thm}(t)$ to a prime theory $\Delta$ in $\operatorname{Var}(t)$ that is disjoint from $\Gamma$. Moreover, if theories are required to be nonempty, then $\Gamma \neq \emptyset$ and so $\Delta$ is proper. Thus putting $a=(\Delta, \operatorname{Var}(t))$ would give $a \in S$ and $a \geq t$; and if $A \in \operatorname{Thm}(u)$ then $\neg A \in \Gamma$ and so $\neg A \notin \operatorname{Thm}(a)$, which implies that $a \not \perp u$. But this contradicts our original supposition. Hence indeed $\operatorname{Thm}(t) \cap \Gamma \neq \emptyset$.

II(iii)': $\alpha \cup(\beta-\gamma)$ is a (nonempty) finite set of real variables if $\alpha, \beta, \gamma$ are.

IV(ii)'(a): Let $t \downarrow \perp u \downarrow$. Then there is an $A \in \operatorname{Thm}(u \downarrow)$ with $\neg A \in \operatorname{Thm}(t \downarrow)$. Now $A \in \operatorname{Thm}(u)$ and $\neg A \in \operatorname{Thm}(t)$, as $\operatorname{Thm}(v \downarrow) \subseteq \operatorname{Thm}(v)$ in general, so $t \perp u$.

IV(ii)'(b): Let $t \perp u \uparrow$, with some $B \in \operatorname{Thm}(u \uparrow)$ having $\neg B \in \operatorname{Thm}(t)$. By definition of $u \uparrow$, there exists $A \in \operatorname{Thm}(u)$ with $A \vdash B$. Hence $\neg B \vdash_{\mathrm{L}} \neg A$ by Antitonicity, so $\neg A \in \operatorname{Thm}(t)$. But $\neg A$ is a formula in $\operatorname{Var}(u)=\operatorname{Var}(t \downarrow)$, so $\neg A \in \operatorname{Thm}(t \downarrow)$, implying $t \downarrow \perp u$.

$\mathbf{V}(\mathbf{v})^{\prime}$ : Let $\rightarrow$ be $^{\mathbf{v w}} \rightarrow$. Suppose $t \perp \vec{u}$, with some $B \in \operatorname{Thm}(\vec{u})$ having $\neg B \in \operatorname{Thm}(t)$. Then there exists $A \in \operatorname{Thm}(u)$ with $A \vdash_{\mathrm{L}^{\mathrm{vw}}} B$. Hence $\neg B \vdash_{\mathrm{L}^{\mathrm{vw}}} \neg A$ by Antitonicity. This ensures $\neg A \in \operatorname{Thm}(\vec{t})$, and hence $\vec{t} \perp u$.

$\mathbf{V}(\mathbf{v i})^{\prime}$ : The new part is that $\vec{t} \vec{u} \leq \overrightarrow{t u}$. This uses the Fusion-Residuation rules, in the form of their consequences in Lemma 6.3(2). Let $B \in \operatorname{Thm}(\vec{t} \vec{u})$. Then there exists $A \rightarrow$ $B \in \operatorname{Thm}(\vec{t})$ with $A \in \operatorname{Thm}(\vec{u})$. Hence there exists $C \in \operatorname{Thm}(t)$ with $C \vdash_{L^{\text {vw }}} A \rightarrow B$, and $D \in \operatorname{Thm}(u)$ with $D \vdash_{\mathrm{L}^{\mathrm{vw}}} A$. Then by Fusion-Monotonicity, $C \circ D \vdash_{\mathrm{L}^{\mathrm{vw}}}(A \rightarrow B) \circ A$. Using T1 of Lemma 6.3(2), this leads to $C \circ D \vdash^{\mathrm{vw}} B$. But by T2 and $C \in \mathrm{Thm}(t)$ we have $D \rightarrow C \circ D \in \operatorname{Thm}(t)$, hence as $D \in \operatorname{Thm}(u), C \circ D \in \operatorname{Thm}(t u)$. From $C \circ D \vdash_{L^{v w}} B$, this gives $B \in \operatorname{Thm}(\overrightarrow{t u})$ as required.

$\mathbf{V}(\mathbf{x}):$ Let $\rightarrow$ be $^{\mathbf{v w}} \overrightarrow{ }$ for $\mathbf{v}, \mathbf{w} \in \operatorname{Var}(t \downarrow)$. To show $\overrightarrow{(t \downarrow)} \geq(\vec{t}) \downarrow$, let $B \in \operatorname{Thm}((\vec{t}) \downarrow)$. Then $\operatorname{Thm}(\vec{t}) \vdash_{\mathrm{L}} B$, so for some $A \in \operatorname{Thm}(t), A \vdash_{\mathrm{L}^{\mathrm{vw}}} B$. Hence by Lemma 3.3, $\vdash_{\mathrm{L}}$ $A(\mathbf{v} / \mathbf{w}) \rightarrow B(\mathbf{v} / \mathbf{w})$.

Let $\operatorname{Var}(A)-\operatorname{Var}(t \downarrow)=\left\{\mathbf{v}_{1}, \ldots, \mathbf{v}_{n}\right\}$, and take new $x_{1}, \ldots, x_{n}$ not occurring in $A$ or $B$. Since the $\mathbf{v}_{i}$ do not occur in $B(\mathbf{v} / \mathbf{w})$, by $\exists$-Elimination we get

$$
\vdash_{\mathrm{L}} \exists x_{n} \ldots \exists x_{1} A\left(x_{1} / \mathbf{v}_{1}, \ldots, x_{n} / \mathbf{v}_{n}\right)(\mathbf{v} / \mathbf{w}) \rightarrow B(\mathbf{v} / \mathbf{w}) .
$$

Let $A^{*}=\exists x_{n} \ldots \exists x_{1} A\left(x_{1} / \mathbf{v}_{1}, \ldots, x_{n} / \mathbf{v}_{n}\right)$. We have shown that $\vdash_{\mathrm{L}} A^{*}(\mathbf{v} / \mathbf{w}) \rightarrow B(\mathbf{v} / \mathbf{w})$. Hence by Lemma 3.3, $A^{*} \vdash^{\mathrm{vw}} B$. But $\vdash_{\mathrm{L}} A \rightarrow A^{*}$ by Lemma 4.1(1), and $A \in \operatorname{Thm}(t)$, so $A^{*} \in \operatorname{Thm}(t)$. Since $A^{*} \in \operatorname{Fml}(\operatorname{Var}(t \downarrow))$, then $A^{*} \in \operatorname{Thm}(t \downarrow)$. Since $A^{*} \vdash_{L^{\text {vw }}} B$, this finally gives $B \in \operatorname{Thm}(\overrightarrow{(t \downarrow)})$ as required.

Lemma 6.6 (CAnonical Truth). In $\mathfrak{A}_{\mathrm{L}}, t \models A$ iff $A \in \operatorname{Thm}(t)$.

Proof. We work through the new inductive cases for $A$. Suppose $t=(\Delta, V)$.

Negation: Let $t \models \neg B$. Put $\Gamma=\left\{C \in \operatorname{Fml}(V): B \vdash_{L} C\right\}$, and $u=(\Gamma, V)$. Then $u \in T$ and $u \equiv t$. But $B \in \operatorname{Thm}(u)$ by the Identity axiom, so $u \models B$ by induction hypothesis, hence $t \perp u$ by the semantics of $\neg$. Thus there is some $C \in \Gamma$ with $\neg C \in \operatorname{Thm}(t)$. By Antitonicity, $\neg C \vdash_{\mathrm{L}} \neg B$, hence $\neg B \in \operatorname{Thm}(t)$ as required.

Conversely, if $\neg B \in \operatorname{Thm}(t)$, then for any $u \equiv t$ with $u=B$ we have $B \in \operatorname{Thm}(u)$ by induction hypothesis, which is enough to show $t \perp u$. Hence $t \models \neg B$.

Disjunction: Let $t=B \vee C$. Now if $B \vee C \notin \Delta$, then there exists a $\Gamma$ extending $\Delta$ that is saturated in $V$ and has $B \vee C \notin \Gamma$. Put $a=(\Gamma, V) \in S$. Then $a \geq t$, so either $a \models B$ or $a \models C$, so by induction hypothesis $B \in \Gamma$ or $C \in \Gamma$. But in either case the Disjunction Introduction axioms lead to the contradiction $B \vee C \in \Gamma$. Hence $B \vee C \in \operatorname{Thm}(t)$ after all. 
Conversely, if $B \vee C \in \operatorname{Thm}(t)$, then for any $a \geq t, B \vee C \in \operatorname{Thm}(a)$, hence $B \in \operatorname{Thm}(a)$ or $C \in \operatorname{Thm}(a)$ as $\operatorname{Thm}(a)$ is prime, so $a \models B$ or $a \models C$ by induction hypothesis. This shows $t=B \vee C$.

Fusion: Let $t \models B \circ C$. Now if $B \vee C \notin \Delta$, there exists a $V$-saturated $\Gamma$ extending $\Delta$ with $B \circ C \notin \Gamma$. Put $a=(\Gamma, V) \in S$. Then $a \geq t$, so the semantics of fusion gives some $u, v$ with $a \geq u v, u \models B$, and $v \models C$. Hence by induction hypothesis $B \in \operatorname{Thm}(u)$ and $C \in \operatorname{Thm}(v)$. By T2 of Lemma 6.3(2), $B \in \operatorname{Thm}(u)$ implies $(C \rightarrow B \circ C) \in \operatorname{Thm}(u)$. Hence $C \in \operatorname{Thm}(v)$ implies $B \circ C \in \operatorname{Thm}(u v) \subseteq \operatorname{Thm}(a)=\Gamma$, a contradiction. Hence $B \circ C \in \operatorname{Thm}(t)$ after all.

Conversely, let $B \circ C \in \operatorname{Thm}(t)$ and take any $a \geq t$. Then $B \circ C \in \operatorname{Thm}(a)$. Put $\Gamma_{B}=\left\{D \in \operatorname{Fml}(V): B \vdash_{\mathrm{L}} D\right\}, \Gamma_{C}=\left\{D \in \operatorname{Fml}(V): C \vdash_{\mathrm{L}} D\right\}, u=\left(\Gamma_{B}, V\right)$, and $v=\left(\Gamma_{C}, V\right)$. Then $B \in \operatorname{Thm}(u)$ and $C \in \operatorname{Thm}(v)$, so $u \models B$ and $v \models C$ by induction hypothesis. It remains to show that $a \geq u v$ to conclude from this that $t \models B \circ C$. But if $E \in \operatorname{Thm}(u v)$, then there is some $D \in \operatorname{Thm}(v)$ with $D \rightarrow E \in \operatorname{Thm}(u)$. Thus $B \vdash_{\mathrm{L}} D \rightarrow E$ and $C \vdash_{\mathrm{L}} D$, so by Fusion-Monotonicity, $B \circ C \vdash_{\mathrm{L}}(D \rightarrow E) \circ D$. This leads by T1 of Lemma 6.3(2) to $B \circ C \vdash_{\mathrm{L}} E$. As $B \circ C \in \operatorname{Thm}(a), E \in \operatorname{Thm}(a)$ as required.

Existential quantifier: Let $t \models \exists x B$. If $\exists x B \notin \Delta$, there exists a $V$-saturated $\Gamma$ extending $\Delta$ with $\exists x B \notin \Gamma$. Put $a=(\Gamma, V) \in S$. Then $a \geq t$, so by the semantics for $\exists$ of Corollary 6.2(2), there exist $u$ and $\mathbf{v} \in D_{u}$ with $u \models B(\mathbf{v} / x)$ and $u \downarrow_{V} \leq a$. By induction hypothesis $B(\mathbf{v} / x) \in \operatorname{Thm}(u)$. Hence by the Existence axiom, $\exists x B \in \operatorname{Thm}(u)$. But $\exists x B \in \operatorname{Fml}(V)$, so then $\exists x B \in \operatorname{Thm}\left(u \downarrow_{V}\right) \subseteq \operatorname{Thm}(a)=\Delta$, a contradiction. Hence $\exists x B \in \operatorname{Thm}(t)$ after all.

Conversely, let $\exists x B \in \operatorname{Thm}(t)$. Take any $a \geq t$. Choose $\mathbf{v} \notin V$ and put $V^{+}=V \cup\{\mathbf{v}\}$. Let $\Gamma=\left\{C \in \operatorname{Fml}\left(V^{+}\right): B(\mathbf{v} / x) \vdash C\right\}$, and $u=\left(\Gamma, V^{+}\right)$. Then $B(\mathbf{v} / x) \in \operatorname{Thm}(u)$, so $u \models B(\mathbf{v} / x)$ by induction hypothesis. It remains to show $u \downarrow_{V} \leq a$ to conclude, via Corollary 6.2(2), that $t \models \exists x B$. But if $C \in \operatorname{Thm}\left(u \downarrow_{V}\right)$, then $B(\mathbf{v} / x) \vdash_{\mathrm{L}} C$ and $C \in$ $\operatorname{Fml}(V)$, so $\mathbf{v}$ is not in $C$. Also $\mathbf{v}$ is not in $B$, so $B(\mathbf{v} / x)(x / \mathbf{v})=B$. Hence $\vdash_{\mathrm{L}} \exists x B \rightarrow C$ by $\exists$-Elimination. From $\exists x B \in \operatorname{Thm}(t)$ we then get $C \in \operatorname{Thm}(t) \subseteq \operatorname{Thm}(a)$, so $C \in \operatorname{Thm}(a)$ as required.

In a canonical model, the semantics of fusion can be simplified:

Corollary 6.7. In $\mathfrak{A}_{\mathrm{L}}, \quad t \models B \circ C$ iff $\exists u, v(t \geq u v$ and $u \models B$ and $v \models C)$.

Proof. Let $t \models B \circ C$ in $\mathfrak{A}_{\mathrm{L}}$, hence $B \circ C \in \operatorname{Thm}(t)$ by the Canonical Truth Lemma just proved. But in the Fusion case of this lemma we defined points $u=\left(\Gamma_{B}, V\right)$ and $v=\left(\Gamma_{C}, V\right)$ of the model with $B \in \operatorname{Thm}(u)$ and $C \in \operatorname{Thm}(v)$, and showed that if $E \in$ $\operatorname{Thm}(u v)$, then $B \circ C \vdash_{\mathrm{L}} E$. Since $B \circ C \in \operatorname{Thm}(t)$, this implies that $\operatorname{Thm}(u v) \subseteq \operatorname{Thm}(t)$, and hence $t \geq u v$. Also $u \models B$ and $v \models C$ by the lemma.

Conversely, if $t \geq u v$ and $u \models B$ and $v \models C$, then transitivity of $\geq$ ensures that $(\forall a \geq$ $t)(a \geq u v$ and $u \models B$ and $v \models C)$, hence $t \models B \circ C$.

We have now completed all the work needed for completeness theorems that axiomatize the logics determined by MQ-models. Theorem 6.4 and Lemmas 6.5 and 6.6, together with the work of the earlier sections allow us to conclude that MQ is characterized by the class of all MQ-models. This completeness result extends to MQX, where X is any subset of a list comprising the postulates of Figure 2 and the axioms of Double-Negation Introduction $A \rightarrow \neg \neg A$, and Contraposition $(A \rightarrow \neg B) \rightarrow(B \rightarrow \neg A)$. Three of the postulates from Figure 2 involve $\neg$ and require new corresponding structure-conditions on $\perp$. The negation-related postulates we can handle are listed in Figure 5. It is left to the interested 


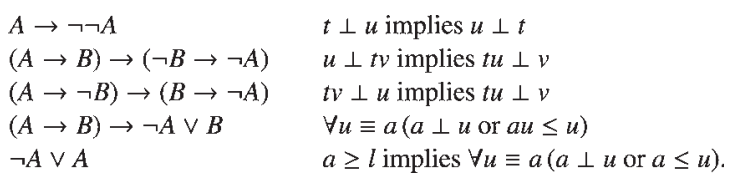

Fig. 5. Negation-Related Postulates and Their $\perp$-Conditions.

reader to show that each of these postulates is valid in models satisfying the corresponding condition, and that, conversely, a canonical model $\mathfrak{A}_{\mathrm{L}}$ satisfies this condition if $\mathrm{L}$ has the postulate.

For all of these logics, completeness also holds for the extension obtained by adding Conjunctive $\exists$-Distribution and restricting to models satisfying IV(i)(d) (see Theorems 4.2 and 6.4(2)). Likewise for the addition of Disjunctive $\forall$-Distribution and IV(i)(b).

As is well known, the Double-Negation Introduction axiom is equivalent to the Contraposition rule over MQ. Indeed, only the Prefixing and Modus Ponens rules are needed to show the Contraposition rule is equivalent to the combination of the Antitonicity rule and the Double-Negation Introduction axiom. The formula analogues of these rules are equivalent over MQ in the presence of Double-Negation Introduction, as indicated by the conditions corresponding to the first three postulates above.

6.1. Identity again. For validity of $\mathrm{I} 4 \mathrm{in}$ general we need a new model condition, since the validity proof we gave for it in Section 3 used the co-theory function $-a$, which has now been replaced by $\perp$. The new condition is

\section{Standard:}

(ix) $\exists u \equiv a(a \not \perp u) \quad$ [equivalently: $(\forall u \equiv t(t \perp u))$ implies $t \notin S$ ].

For languages with a distinguished identity predicate $\approx$, we define an $M Q$-model with identity to be an MQ-model that satisfies VI(i) and also this I(ix). In such a model, we get $t \models i \not \varpi i \rightarrow A$ for any $A \in \operatorname{Sen}(t)$. For if not, there exists $u \equiv t$ with $u \models i \not \approx i$ while $t u \forall A$. Then by Truth Saturation, there exists $a \geq t u$ with $a \forall A$. Hence by I(iii), there is some $b \in S$ with $b \geq u$ (and $a \geq t b$ ). Then by the new I(ix), there is some $v \equiv b$ with $b \not \downarrow v$. Since $b \models i \not \approx i$ by Truth Extension, the semantics of $\neg$ then implies $v \not \forall i \approx i$, which contradicts the fact that self-identities are unfalsifiable. This is enough to ensure that I4 is valid in the present semantics. Note that the argument used the second part of model condition I(iii), the part whose proof in a canonical model depends on I4 itself (Remark 3.6).

For a logic $\mathrm{L}$ with the identity axioms, the canonical model $\mathfrak{A}_{\mathrm{L}}$ satisfies I(ix). To see this, take any $a \in S$ in such a model, with $\operatorname{Var}(a)=V$. Take any $\mathbf{v} \in V$, let $\Gamma=\{A \in \operatorname{Fml}(V)$ : $\left.\mathbf{v} \approx \mathbf{v} \vdash_{L} A\right\}$ and put $u=(\Gamma, V) \in T$. Then $a \not \perp u$ as desired, since otherwise there would be some $A$ with $\mathbf{v} \approx \mathbf{v} \vdash_{\mathrm{L}} A$ and $\neg A \in \operatorname{Thm}(a)$. But $\neg A \vdash_{\mathrm{L}} \mathbf{v} \not \approx \mathbf{v}$ by Antitonicity, hence $\mathbf{v} \not \approx \mathbf{v} \in \operatorname{Thm}(a)$. Since I1 and I4 yield $\vdash_{\mathrm{L}} \mathbf{v} \not \approx \mathbf{v} \rightarrow B$, this puts every $V$-formula into $\operatorname{Thm}(a)$, contradicting the fact that $\operatorname{Thm}(a)$ is proper.

These facts allow us to establish that

- $\mathrm{MQ}^{\approx}$, the smallest logic in the language with $\approx$ that has the identity axioms, is characterized by the class of all MQ-models with identity.

Again, these results extend to the systems $\mathrm{MQX} \approx$, and to their extensions by Conjunctive $\exists$-Distribution and Disjunctive $\forall$-Distribution, with their corresponding model conditions. 
Now the proof of conservativity of adding $\supset$ in Theorem 5.2 depended on the presence of the model condition IV(i)(d), corresponding to the Conjunctive $\exists$-Distribution axiom (see Theorem 4.2). We write $\mathrm{L}^{\exists}$ for the extension of a logic $\mathrm{L}$ by this axiom. Let $X$ be any subset of the list comprising the negation-free postulates of Figure 2 and the negationrelated postulates of Figure 5. Then by the method of Section 5 we get

- $\mathrm{H}\left(\mathrm{MQX}^{\exists}\right)^{\approx}$ is a conservative extension of $\left(\mathrm{MQX}^{\exists}\right)^{\approx}$.

Remark 6.8 ( $\wedge$ - $\exists$-Distribution Is Essential). The Conjunctive $\exists$-Distribution postulate is essential to this result, since it is derivable in $\mathrm{HL}$ for any logic $\mathrm{L}$, so HL is not conservative over $\mathrm{L}$ if $\mathrm{L}$ does not contain the postulate. To see this, for given formulas $A, B$ with $x$ not free in $A$, take any real $\mathbf{v}$ that does not occur in $A$ or $B$. Then $(A \wedge B)(\mathbf{v} / x))=$ $A \wedge(B(\mathbf{v} / x))$, so by the Existence axiom,

$$
\vdash_{\mathrm{HL}} A \wedge(B(\mathbf{v} / x)) \rightarrow \exists x(A \wedge B) .
$$

Hence by $\wedge$-Residuation (and commutativity of $\wedge$ ),

$$
\vdash \mathrm{HL} B(\mathbf{v} / x) \rightarrow(A \rightarrow \exists x(A \wedge B)) .
$$

Since $\mathbf{v}$ does not occur in $A \rightarrow \exists x(A \wedge B)$, the $\exists$-Elimination rule then gives

$$
\vdash_{\mathrm{HL}} \exists x B \rightarrow(A \rightarrow \exists x(A \wedge B)),
$$

which leads by $\wedge$-Residuation again to

$$
\vdash_{\mathrm{HL}} A \wedge \exists x B \rightarrow \exists x(A \wedge B) .
$$

REMARK 6.9 (The PLACE OF I3 AND I4). The identity axiom I3: $\mathbf{v} \approx \mathbf{v} \rightarrow(\mathbf{v} \approx \mathbf{v} \rightarrow$ $\mathbf{v} \approx \mathbf{v})$ is redundant over MQ, since $(\mathbf{v} \approx \mathbf{v} \circ \mathbf{v} \approx \mathbf{v}) \rightarrow \mathbf{v} \approx \mathbf{v}$ is an instance of I1, and $\mathrm{I} 3$ follows from this by the second Fusion-Residuation rule. That works also in RQ, where the fusion connective is definable.

Remark 3.6 pointed out that $\mathrm{I} 4$ is not needed in logics containing the Assertion axiom $A \rightarrow((A \rightarrow B) \rightarrow B)$, which corresponds to the commutative model condition $t u=u t$. The logic characterized by the class of all commutative MQ-models with identity is axiomatizable by adding just I1 and $\mathrm{I} 2$ to $\mathrm{MQ}+$ Assertion. But whereas I4 was seen to be derivable from I1 over BQ+Assertion, this derivation used Double-Negation Elimination and does not carry through over MQ+Assertion. For logics with the weaker Antitone or Contrapositive Negation, inclusion of I4 requires the additional model condition I(ix). Inclusion of I4 is necessary for logics that lack Assertion.

\$. Conservatively adding $\approx$. This section describes a proof that a predicate satisfying the main identity axioms I1 and I2 can be conservatively added to MQ- $\exists$, the $\exists$-free fragment of MQ. The proof will also directly show the conservativity of adding $\supset$ to this fragment, and to some of its extensions, including MQ- $\exists+$ Assertion.

We assume now that our language has the primitives $\tau, \wedge, \vee, \neg, \rightarrow, \circ, \forall$; but not $\exists$. A $\operatorname{logic} \mathrm{L}$ for this language has all the postulates of MQ except for those involving $\exists$. MQ $-\exists$ is the smallest such logic.

The idea of the conservativity proof is to construct a characteristic algebraic model $\mathcal{M}_{\mathrm{L}}$ for $\mathrm{L}$ by the standard Lindenbaum method, and then embed this Lindenbaum algebra into its ideal completion $\mathcal{M}_{\mathrm{L}}^{+}$. This embedding preserves the interpretation of sentences, and gives an order-complete algebraic model falsifying the nontheorems of L. Self-identities are interpreted as the top element of the completion, and other identities as the bottom 
element. The order-completeness is used to interpret $\forall x A$, intuitively as the infinite conjunction of all its specifications $A(i / x)$. This defines a model satisfying I1 and I2, and any further axioms whose truth is preserved by ideal completion. In addition $\mathcal{M}_{\mathrm{L}}^{+}$, as the ideal completion of a distributive lattice, is in fact a Heyting algebra, so it can be used to show the conservativity of adding $\supset$. We spell out some details:

Definition 7.1. A basic algebra $\mathcal{Q}=(Q, \leq, \sqcap, \sqcup, \Rightarrow, \bullet,-, 1)$ comprises:

- A partial order $\leq$ on the set $Q$, making it into a distributive lattice with meet $\sqcap$ and join $\sqcup$ operations that will be used to interpret the connectives $\wedge$ and $\vee$.

- Binary operations $\Rightarrow$ and $\bullet$ on $Q$ interpreting the connectives $\rightarrow$ and $\circ$. These satisfy:

$$
\begin{aligned}
& a \leq a^{\prime} \text { and } b \leq b^{\prime} \text { implies } a \bullet b \leq a^{\prime} \bullet b^{\prime} ; \\
& a \bullet b \leq c \quad \text { iff } \quad a \leq b \Rightarrow c ; \\
& a \bullet(b \sqcup c) \leq(a \bullet b) \sqcup(a \bullet c) .
\end{aligned}
$$

- A unary operation - : $Q \rightarrow Q$, interpreting $\neg$, that is antitone: $a \leq b$ implies $-b \leq-a$.

- A distinguished element 1 , to interpret $\boldsymbol{\tau}$. This satisfies $1 \bullet a=a$, and $a \leq b$ iff $1 \leq a \Rightarrow b$.

Given a set $I$ (of individuals), as previously we add the members of $I$ to our language as self-designating constants. A sentence is a formula that may contain these constants but has no free variables.

An algebraic model $\mathcal{M}=(\mathcal{Q}, I, \theta)$ consists of a basic algebra $\mathcal{Q}$, a nonempty set $I$ of individuals, and a valuation $\theta$, which is a function assigning to each sentence $A$ an element $\theta A$ of $\mathcal{Q}$, such that

- $\theta(A \wedge B)=\theta A \sqcap \theta B, \theta(A \vee B)=\theta A \sqcup \theta B, \theta(A \rightarrow B)=\theta A \Rightarrow \theta B$, $\theta(A \circ B)=\theta A \bullet \theta B$

- $\theta(\neg A)=-\theta A, \quad \theta \boldsymbol{\tau}=1$

- $\theta(\forall x A)=\prod_{i \in I} \theta(A(i / x))$,

where $\prod$ denotes meet (greatest lower bound). Note that we do not require that every subset of $Q$ has a meet, but only that for $\theta$ to qualify as a valuation, every set of the form $\{\theta(A(i / x)): i \in I\}$ must have a meet, and it must be equal to $\theta(\forall x A)$.

A sentence $A$ is true in $\mathcal{M}$, written $\mathcal{M} \models A$, if $1 \leq \theta(A)$. For relevant implications, this means that $\mathcal{M} \models A \rightarrow B$ iff $\theta A \leq \theta B$. A real formula $A\left(\mathbf{v}_{1}, \ldots, \mathbf{v}_{n}\right)$ is true in $\mathcal{M}$ if for any $i_{1}, \ldots, i_{n} \in I, \mathcal{M} \models A\left(i_{1} / \mathbf{v}_{1}, \ldots, i_{n} / \mathbf{v}_{n}\right)$. Standard algebraic reasoning shows that all real axioms of MQ $-\exists$ are true in $\mathcal{M}$, and that the rules of MQ- $\exists$ preserve this truth.

For the Lindenbaum construction, let $Q_{\mathrm{L}}$ be the set of all equivalence classes $|A|$ of real formulas $A$, where $|A|$ is the set of all real $B$ such that $\vdash_{\mathrm{L}}(A \rightarrow B) \wedge(B \rightarrow A)$. $Q_{\mathrm{L}}$ is a distributive lattice under the partial order defined by $|A| \leq|B|$ iff $\vdash_{\llcorner} A \rightarrow B$, with $|A| \sqcap|B|=|A \wedge B|$ and $|A| \sqcup|B|=|A \vee B|$. It becomes a basic algebra $\mathcal{Q}_{\mathrm{L}}$ by putting $|A| \Rightarrow$ $|B|=|A \rightarrow B|,|A| \bullet|B|=|A \circ B|$, and $1=|\tau|$. If $\mathcal{R}$ is the set of real variables, then the canonical algebraic model for $\mathrm{L}$ is $\mathcal{M}_{\mathrm{L}}=\left(\mathcal{Q}_{\mathrm{L}}, \mathcal{R}, \theta_{\mathrm{L}}\right)$, where $\theta_{\mathrm{L}}(A)=|A|$ for each real formula $A$, viewed as a sentence in which each $\mathbf{v}$ from $\mathcal{R}$ is a self-designating name.

The proof that this $\theta_{\mathrm{L}}$ is a valuation is standard, but we review the condition for $\forall$. First, the Specification axiom ensures that $|\forall x A| \leq|A(\mathbf{v} / x)|$ for all $\mathbf{v} \in \mathcal{R}$. But if $|B| \leq$ 
$\mid\left(A(\mathbf{v} / x) \mid\right.$ for all $\mathbf{v}$, then in general $\vdash_{\mathrm{L}} B \rightarrow A(\mathbf{v} / x)$. In particular, this holds for $\mathbf{v}$ not in $B$ or in $A$ itself, from which $\vdash \forall x(B \rightarrow A)$ follows by the Generalization rule. Hence $\vdash_{\mathrm{L}} B \rightarrow \forall x A$ by the Relevant $\forall$-Distribution axiom, leading to $|B| \leq|\forall x A|$. This shows that $|\forall x A|=\prod_{\mathbf{v} \in \mathcal{R}}|A(\mathbf{v} / x)|$, that is that $\theta_{\mathrm{L}}(\forall x A)=\prod_{\mathbf{v} \in \mathcal{R}} \theta_{\mathrm{L}}(A(\mathbf{v} / x))$.

The ideal completion of $\mathcal{Q}_{\mathrm{L}}$ is the structure

$$
\mathcal{Q}_{\mathrm{L}}^{+}=\left(Q_{\mathrm{L}}^{+}, \subseteq, \cap, \sqcup, \Rightarrow, \bullet,-, 1^{+}\right),
$$

defined as follows. Its members are the ideals of $\mathcal{Q}_{\mathrm{L}}$, which will be denoted $\mathcal{A}, \mathcal{B}$. Recall that an ideal is a subset of $Q_{\mathrm{L}}$ that is closed under binary joins $a \sqcup b$ and closed downward under the partial order $\leq$. $Q_{\mathrm{L}}^{+}$contains the principal ideal $\downarrow a=\{b: b \leq a\}$ generated by each $a \in Q$. It is a complete lattice under the inclusion ordering $\subseteq$, with the meet $\prod_{j \in J} \mathcal{A}_{j}$ of a set of ideals being its intersection $\bigcap_{J} \mathcal{A}_{j}$, and the join given by

$$
\bigsqcup_{j \in J} \mathcal{A}_{j}=\bigcup\left\{\downarrow\left(a_{1} \sqcup \cdots \sqcup a_{n}\right): a_{1}, \ldots, a_{n} \in \bigcup_{J} \mathcal{A}_{j}\right\} .
$$

The other operations of $\mathcal{Q}_{\mathrm{L}}^{+}$are given by

$$
\begin{aligned}
\mathcal{A} \Rightarrow \mathcal{B} & =\{c: \forall a \in \mathcal{A} \exists b \in \mathcal{B}(c \leq a \Rightarrow b)\} \\
\mathcal{A} \bullet \mathcal{B} & =\bigcup\{\downarrow(a \bullet b): a \in \mathcal{A} \text { and } b \in \mathcal{B}\} \\
-\mathcal{A} & =\bigcap_{a \in \mathcal{A}} \downarrow(-a) \\
1^{+} & =\downarrow 1 .
\end{aligned}
$$

$\mathcal{Q}_{\mathrm{L}}^{+}$is a basic algebra, and the map $a \mapsto \downarrow a$ is an injection of $\mathcal{Q}_{\mathrm{L}}$ into $\mathcal{Q}_{\mathrm{L}}^{+}$that preserves all the basic algebra operations and preserves and reflects the partial orders, that is $a \leq b$ iff $\downarrow a \subseteq \downarrow b$ (there is a discussion in Restall, 2000, Section 9.2, that gives a good deal of the detail of these claims). Importantly, this map also preserves any meets $\prod_{J} a_{j}$ that happen to exist in $\mathcal{Q}_{\mathrm{L}}$. For, by definition, $b \leq \prod_{J} a_{j}$ iff $b \leq a_{j}$ for all $j \in J$, which means that $\downarrow \prod_{J} a_{j}=\bigcap_{J} \downarrow a_{j}$

A model $\mathcal{M}_{\mathrm{L}}^{+}=\left(\mathcal{Q}_{\mathrm{L}}^{+}, \mathcal{R}, \theta^{+}\right)$for the language including the identity predicate is obtained by defining the value $\theta^{+}(A)$ inductively on the length of sentence $A$, as follows:

- $\theta^{+}(\mathbf{v} \approx \mathbf{v})=Q_{\mathrm{L}}$, the largest ideal of $\mathcal{Q}_{\mathrm{L}}$.

- For $\mathbf{v} \neq \mathbf{w}, \theta^{+}(\mathbf{v} \approx \mathbf{w})=\emptyset$, the smallest ideal.

- For $A$ an atomic sentence other than an identity, $\theta^{+}(A)=\downarrow|A|$.

- For the inductive cases, $\theta^{+}(A)$ is defined by the conditions specifying that it is a valuation, that is $\theta^{+}(A \wedge B)=\theta^{+} A \cap \theta^{+} B, \theta^{+}(\neg A)=-\theta^{+} A$, and so forth. In particular $\theta^{+}(\forall x A)=\bigcap_{\mathbf{v} \in \mathcal{R}} \theta^{+}(A(\mathbf{v} / x))$.

Thus $\theta^{+}$is the unique valuation determined by the given values on atomic sentences.

LEMMA 7.2. If $A$ is any $\approx$-free sentence, $\theta^{+}(A)=\downarrow \theta_{\mathrm{L}}(A)$. Hence $\mathcal{M}_{\mathrm{L}} \models A$ iff $\mathcal{M}_{\mathrm{L}}^{+} \models A$.

Proof. For the first statement, if $A$ is atomic, the result holds by definition of $\theta^{+}$and $\theta_{\mathrm{L}}$. The inductive cases follow because $a \mapsto \downarrow a$ preserves the basic algebra operations and any existing meets. For example

$$
\downarrow \theta_{\mathrm{L}}(\forall x A)=\downarrow \prod_{\mathbf{v} \in \mathcal{R}} \theta_{\mathrm{L}}(A(\mathbf{v} / x))=\bigcap_{\mathbf{v} \in \mathcal{R}} \downarrow \theta_{\mathrm{L}}(A(\mathbf{v} / x))=\bigcap_{\mathbf{v} \in \mathcal{R}} \theta^{+}(A(\mathbf{v} / x))=\theta^{+}(\forall x A) .
$$

For the second statement, $1 \leq \theta_{\mathrm{L}}(A)$ iff $\downarrow 1 \subseteq \downarrow \theta_{\mathrm{L}}(A)$ iff $1^{+} \subseteq \theta^{+}(A)$. 
THEOREM 7.3. The identity axioms II and I2 can be conservatively added to $M Q-\exists$.

Proof. Let $\mathrm{L}=\mathrm{MQ}-\exists$, and take $M_{\mathrm{L}}$ and $\mathcal{M}_{\mathrm{L}}^{+}$as above. If $B$ is any $\approx$-free formula such that $\nvdash_{\mathrm{L}} B$, then $\mathcal{M}_{\mathrm{L}} \forall B$, because $\nvdash_{\mathrm{L}} \tau \rightarrow B$ and so $1 \not \leq \theta_{\mathrm{L}}(B)$ in $\mathcal{M}_{\mathrm{L}}$. Hence by the above lemma, $\mathcal{M}_{\mathrm{L}}^{+} \not=B$.

Since $\mathcal{Q}_{\mathrm{L}}^{+}$is a basic algebra, $\mathcal{M}_{\mathrm{L}}^{+}$is a model of MQ- $\exists$ in the language with $\approx$. To complete the theorem then, it suffices to show it is also a model of the two identity axioms.

For any sentence $A, \theta^{+}(A) \subseteq Q_{\mathrm{L}}^{+}=\theta^{+}(\mathbf{v} \approx \mathbf{v})$, so $\mathcal{M}_{\mathrm{L}}^{+} \models A \rightarrow \mathbf{v} \approx \mathbf{v}$, that is $\mathcal{M}_{\mathrm{L}}^{+}$is a model of I1. For any instance $\mathbf{v} \approx \mathbf{w} \wedge A \rightarrow A^{\prime}$ of I2, note first that if $\mathbf{v}=\mathbf{w}$, then $A^{\prime}=A$ and this is just an instance of the valid Conjunction Elimination axiom. But if $\mathbf{v} \neq \mathbf{w}$, then $\theta^{+}(\mathbf{v} \approx \mathbf{w} \wedge A)=\emptyset \subseteq \theta^{+}\left(A^{\prime}\right)$, so $\mathcal{M}_{\mathrm{L}}^{+}$is a model of $\mathrm{I} 2$.

In this conservativity result, MQ- $\exists$ can be replaced by a number of its extensions got by adding various postulates. All that is required for the proof is that validity of the postulate be preserved in passing from $\mathcal{M}_{\mathrm{L}}$ to $\mathcal{M}_{\mathrm{L}}^{+}$. In this way it can be shown that I1 and I2 can be conservatively added to MQ $-\exists+X$, where $\mathrm{X}$ is any subset of a list comprising the negation-free postulates of Figure 2 and the negation-related postulates of Figure 5. Verification of this is left to the reader.

Now it is well known that the ideal completion of a distributive lattice is a Heyting algebra (a result of M. H. Stone, see Birkhoff, 1967, p. 129). Indeed in $\mathcal{Q}_{\mathrm{L}}^{+}$we can define a binary operation $\ni$ on ideals by

$$
\mathcal{B} \ni \mathcal{C}=\{a:(\forall b \in \mathcal{B}) a \sqcap b \in \mathcal{C}\},
$$

and show that $\mathcal{A} \cap \mathcal{B} \subseteq \mathcal{C}$ iff $\mathcal{A} \subseteq \mathcal{B} \ni \mathcal{C}$. So putting $\theta^{+}(B \supset C)=\theta^{+}(B) \ni \theta^{+}(C)$ produces a model validating the $\wedge$-Residuation rules (5.2) for $\supset$. This leads to a direct proof that

\section{Heyting implication is conservative over all logics $M Q-\exists+X$ of the previous paragraph.}

This application of ideal completions to conservativity results is rather limited. Apparently validity of Double Negation Elimination is not preserved from $\mathcal{M}_{\mathrm{L}}$ to $\mathcal{M}_{\mathrm{L}}^{+}$. Even if $\mathcal{Q}_{\mathrm{L}}$ has $--a=a$, then in $\mathcal{Q}_{\mathrm{L}}^{+}$we can only show that $--\mathcal{A}=\bigcap\{\downarrow a: \mathcal{A} \subseteq \downarrow a\}$, and this might be a proper superset of $\mathcal{A}$. Also, it seems that $\mathcal{M}_{\mathrm{L}}^{+}$need not validate Disjunction $\forall$-Distribution, and that the map $a \mapsto \downarrow a$ need not preserve existing joins, so the method cannot be applied to postulates for $\exists$ at all. There is another construction, the MacNeille completion of a lattice, that does preserve any existing joins and meets, but it does not preserve $\wedge \vee$-Distribution, so is unsuitable for the kind of logics we are discussing.

§8. Summary. The semantic characterizations and conservativity results that have been proved in this paper can be catalogued as follows.

(1) In the language with primitives $\tau, \wedge, \neg, \rightarrow, \forall$, and with $\vee$ and $\exists$ defined, let $L$ be any of the logics BQX ${ }^{\tau}$, defined by the postulates for BQ listed in Figure 1, the $\tau$-postulates (see Figure 4), and any subset X of the postulates listed in Figure 2. Let $\mathrm{L} \approx$ be the expansion of $\mathrm{L}$ by an identity predicate having axioms I1-I4.

(a) $\mathrm{L} \approx$ is characterized by the class of all stratified models that satisfy all the conditions corresponding to the postulates in X, the condition I(viii) for $\tau$ (see (2.2)), and the identity condition VI(i): $i \approx_{t} j$ iff $t={ }^{i j} \vec{t}$. [Theorem 3.5] 
(b) $\mathrm{L} \approx$ is also characterized by the class of all models as in (a) that satisfy IV(i)(d): $u \geq t \downarrow$ implies $\exists u^{+} \geq t\left(u^{+} \downarrow=u\right)$. [Theorem 4.2]

(c) Heyting implication can be conservatively added to $\mathrm{L} \approx$, that is $\mathrm{HL} \approx$ is a conservative extension of $\mathrm{L} \approx$. [Theorem 5.2]

(2) In the language with primitives $\tau, \wedge, \vee, \neg, \rightarrow, \circ, \forall, \exists$, let $\mathrm{L}$ be any of the logics MQX, where MQ is defined in Figure 4 (which includes the $\tau$-postulates), and $X$ is any subset of the list comprising the negation-free postulates of Figure 2 and the negation-related postulates of Figure 5 . Let $\mathrm{L}^{\exists}$ be $\mathrm{L}$ plus Disjunctive $\exists$-Distribution, and $\mathrm{L}^{\forall}$ be $\mathrm{L}$ plus Conjunctive $\forall$-Distribution.

Recall the notion of MQ-model and MQ-model with identity from Section 6.

(a) L is characterized by the class of all MQ-models that satisfy all the conditions corresponding to the postulates in X.

(b) $\mathrm{L}^{\exists}$ is characterized by the class of all models as in (a) that satisfy IV(i)(d).

(c) $\mathrm{L}^{\forall}$ is characterized by the class of all models as in (a) that satisfy condition IV(i)(b) from Section 2.

(d) Heyting implication is conservative over $\left(\mathrm{L}^{\exists}\right) \approx$, and over $\left(\mathrm{L}^{\forall \exists}\right) \approx$.

(3) In the language with $\tau, \wedge, \vee, \neg, \rightarrow, \circ, \forall$, but without $\exists$, let $\mathrm{L}$ be any of the logics MQ $-\exists+X$, where MQ $-\exists$ is defined by all the postulates of MQ except those involving $\exists$, and $X$ is any subset of a list comprising the negation-free postulates of Figure 2 and the negation-related postulates of Figure 5.

(a) Heyting implication is conservative over $\mathrm{L}$.

(b) The identity axioms I1 and I2 are conservative over L.

The most significant unanswered question concerns the conservativity of Heyting implication over the nonidentity logics BQX ${ }^{\tau}$, especially $\mathrm{RQ}^{\boldsymbol{\tau}}$. Having shown that HBQX ${ }^{\tau} \approx$ is a conservative extension of $\mathrm{BQX}{ }^{\tau} \approx$, the question is reduced to the conservativity of adding identity: is $\mathrm{BQX}{ }^{\boldsymbol{\tau}} \approx$ a conservative extension of $\mathrm{BQX}^{\boldsymbol{\tau}}$ ? The solution may require new techniques, possibly a proof-theoretic analysis.

\section{BIBLIOGRAPHY}

Anderson, A. R., Belnap, N. D., Jr., \& Dunn, J. M. (1992). Entailment: The Logic of Relevance and Necessity, Vol. II. Princeton University Press.

Birkhoff, G. (1967). Lattice Theory (third edition). American Mathematical Society, Providence, Rhode Island.

Dunn, J. M. (1993). Star and perp: Two treatments of negation. Philosophical Perspectives, 7, 331-357.

Fine, K. (1974). Models for entailment. Journal of Philosophical Logic, 3, 347-372. Reprinted in Anderson et al. (1992, §51).

Fine, K. (1988). Semantics for quantified relevance logic. Journal of Philosophical Logic, 17, 22-59. Reprinted in Anderson et al. (1992, §53).

Goldblatt, R. I. (1974). Semantic analysis of orthologic. Journal of Philosophical Logic, 3, 19-35.

Mares, E. D. (1992). Semantics for relevance logic with identity. Studia Logica, 51(1), $1-20$. 
Restall, G. (1998). Displaying and deciding substructural logics 1: Logics with contraction. Journal of Philosophical Logic, 27, 179-216.

Restall, G. (2000). An Introduction to Substructural Logics. Routledge, London.

Routley, R., \& Meyer, R. K. (1973). The semantics of entailment. In Leblanc, H., editor. Truth, Syntax and Modality. North-Holland, Amsterdam, pp. 199-243.

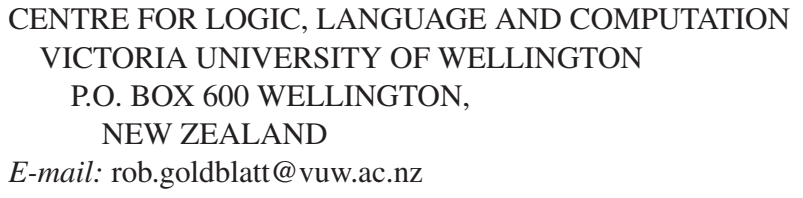

E-mail: rob.goldblatt@vuw.ac.nz 